\title{
An iterative near-boundary reconstruction strategy for unstructured finite volume method
}

\author{
Zedong Chen ${ }^{\mathrm{a}, \mathrm{b}}$, Fan Zhang ${ }^{\mathrm{c}}$, Jun Liu ${ }^{\mathrm{b}, \mathrm{d}, *}$, Biaosong Chen ${ }^{\mathrm{a}, \mathrm{b}}$ \\ ${ }^{a}$ Department of Engineering Mechanics, Dalian University of Technology, Dalian 116023, Liaoning, China \\ ${ }^{b}$ State Key Laboratory of Structural Analysis for Industrial Equipment, Dalian University of Technology, Dalian 116023, Liaoning, China \\ ${ }^{c}$ Centre for mathematical Plasma-Astrophysics, Department of Mathematics, KU Leuven, Celestijnenlaan 200B, 3001 Leuven, Belgium \\ ${ }^{d}$ School of Aeronautics and Astronautics, Dalian University of Technology, Dalian 116024, Liaoning, China
}

\section{A R T ICLE INFO}

\section{Article history:}

Keywords: near-boundary, gradien$\mathrm{t}$ reconstruction, unstructured FVM, vertex-based WLSQ, iterative strategy, vectorial limiter

\begin{abstract}
A B S T R A C T
Near-boundary approximation of the second-order cell-centered unstructured finite volume method is investigated and improved to avoid accuracy loss. In this work, an iterative strategy is proposed, combining with a vertex-based weighted least squares gradient reconstruction method, which is more accurate and efficient than several conventional methods. In the present approach, the solutions at boundary surfaces and the gradients in boundary cells are iteratively updated, to minimize overall numerical errors. In the meantime, a vectorial limiter is implemented to guarantee the correctness of the iterative process, and physical properties of boundaries are taken into account to correctly attain physical results. A series of numerical test cases show that the present method significantly reduces the error of near-boundary approximations. Moreover, since the iterative scheme is only activated for near-boundary solutions, the minor extra computational effort does not damage the overall performance of the solver.
\end{abstract}

(C) 2020 Elsevier Inc. All rights reserved.

\section{Introduction}

Despite the fact that unstructured high-order finite volume methods (FVM) have been very promising and popular in the computational fluid dynamics (CFD) research community [1, 2, 3, 4], the second-order FVM is still a prominent and dominating numerical method for simulating complex flows in both scientific and industrial scenarios, and it is still developing with much effort, aiming at further improvements regarding both accurate approximation and/or computational efficiency. An extensive overview of unstructured FVM discretizations and solvers was given by Mavriplis [5]. The major advantages of the second-order FVM are its efficiency and robustness. However, it is

\footnotetext{
${ }^{*}$ Corresponding author.

e-mail: chenzd_dut@163.com (Zedong Chen), fan.zhang@kuleuven. be,zhangfan.zf@mail.ru (Fan Zhang), liujun65@dlut.edu.cn (Jun Liu), chenbs@dlut.edu.cn (Biaosong Chen)
} 
worth noted that, in terms of accuracy, the conventional second-order FVM may be surpassed by the the discontinuous Galerkin method (DGM) [6], and the Flux-Reconstruction method [7], which can be viewed as a type of nodal DGM. In order to improve the performance of FVM, the spatial reconstruction step is essential for achieving second-order (or higher-order) accurate approximation based on cell-averaged FVM solutions [8, 9, 10, 11, 12, 13].

For the cell-centered (CC) FVM, one class of gradient reconstruction methods is the (vertex-based) node-averagingGreen-Gauss (NA-GG) schemes, which have been extensively investigated $[14,15,16,17,18,19]$ due to their simplicity and efficiency. NA-GG schemes usually perform pseudo-laplacian (PL) procedure $[14,16,17,18]$ to calculate vertex solutions at first, and then cell gradients are calculated by the GG procedure [20], which performs the numerical integral over cell faces. As is well known, the conventional GG method loses linear exactness on irregular grids, and thus improvements are necessary $[11,21]$. Another class of gradient reconstruction methods is cell-based weightedleast-squares (WLSQ) methods. The idea of WLSQ methods is straightforward [22], and the accuracy and stability of WLSQ methods depends on their stencils which provide spatial solution data. It is well known that the face neighbour stencil is usually not sufficient for stability and accuracy, especially on distorted grids. Therefore, in order to improve the accuracy of WLSQ methods, the full-augmentation (FA) stencil or smart-augmentation (SA) stencil is effective $[8,10]$, which provide more spatial information. As a state-of-the-art approach, the F-decreasing augmentation combining with a symmetric augmentation results robust and efficient gradient stencils, improving the performance of the (W)LSQ reconstruction even on highly distorted quadrilateral and triangular grids [13]. In the meantime, the WL$\mathrm{SQ}(\mathrm{G})$ scheme [23] takes the effects of face area and grid distortion into account, effectively improving the accuracy of gradient reconstructions on high-aspect-ratio grids close to curving boundaries.

Another novel approach is the averaged-gradient method invented by Zhang [24, 25]. In this method, gradients are computed first at each grid vertex by using the vertex-based WLSQ (VWLSQ) method with cell-averaged solutions in the cells surrounding the vertex, and then cell-gradients are obtained by averaging the vertex gradients over all the vertexes in the cell. This approach reduces the storage requirement for the WLSQ coefficients and the cost of gradient reconstructions on unstructured grids. Especially, on tetrahedral grids, the amount of grid vertexes can be smaller by a factor of 6 than the amount of grid cells, and thus the advantage of this novel gradient reconstruction method can be more significant. Moreover, the vertex solutions are not used and thus are not subject to the clipping procedure which is a necessity while using PL methods $[14,16,17,18]$. Zhang has shown that the averaged gradients are more accurate than the Green-Gauss gradients computed from the vertex solutions, and the resulting scheme leads to superior accuracy and efficiency for inviscid problems on several typical grid types [24, 25]. This method is easy to be extended for calculating interface gradients used to simulate viscous flows, as shown by Nishikawa and White [12]. Moreover, Nishikawa and White directly employ the face-averaged gradients to linearly reconstruct the solution from both cells across the face to the face centroid, and thus their method shows the advantage of reducing memory requirement for the gradients and the residual stencil which improves the efficiency of implicit temporal solutions $[12,26]$.

In general, the linearly-exact gradient reconstruction methods have been extensively investigated and developed, and in fact these methods are also commonly applied for compressible and incompressible flows simulations. Especially, the present work is done based on an in-house solver implementing these methods. However, to the authors' knowledge, only a little attention, e.g. Ref. [27, 28], has been paid to the near-boundary performance of gradient reconstruction schemes.

Moreover, in order to improve the near-boundary approximation, besides the gradient reconstruction procedure, how to impose boundary conditions (BCs) is also a critical issue. Previous research indicates that the weak-BCs, which are imposed through numerical fluxes with states specified by physical boundary conditions, perform better in terms of convergence and accuracy [29,30]. More specifically, there are two approaches to calculate the boundary fluxes, namely the Weak-Riemann and Weak-Prescribed approaches [31]. While using the Weak-Riemann method $[21,32,33]$, the right states (of numerical flux functions) are specified by physical boundary conditions, and then the boundary fluxes at boundary face quadrature points can be calculated in a unified manner as calculating inner numerical fluxes, which is one of the advantages. The Weak-Prescribed method imposes constraints on the boundary face solutions, and then directly uses the solutions to calculate boundary fluxes $[34,35,36]$. Therefore, accurate right states or boundary face solutions are actually essential for the overall performance of near-boundary approximation. In this work, the Weak-Prescribed method is applied since the boundary face solutions will be calculated together with the boundary gradients. To the authors' knowledge, the boundary face solutions are mostly given by simply copying the value from the boundary cell centroid to the boundary faces and modifying the values according to BCs (first-order extrapolation) [35, 36]. In this way, the solutions are not accurate for linear functions, and thus might spoil 
the advantage of using a linear-exact gradient reconstruction method. Therefore, achieving second-order accurate solutions in the cells and at the boundary faces altogether may be helpful to guarantee the linear exactness of the second-order FVM.

Lee et al. [37] designed an iterative strategy to improve the near-boundary approximation of the Frank's gradient reconstruction method [15, 17]. In this work, a different iterative near-boundary spatial (solution and gradient) reconstruction method is implemented for improving the near-boundary approximation of the VWLSQ-averaged-gradient method [24, 25], and especially a vectorial limiter is introduced to guarantee the correctness of the iterative solutions. It should be noted that for smooth solutions on regular isotropic meshes, the limiter is unnecessary. However, the limiter may be helpful to avoid violating the geometrical monotonicity which is critical on high-aspect-ratio curved grids [23], or numerical oscillation caused by discontinuities interacting with boundaries. The present iterative procedure in fact calculates boundary gradients and solutions as a whole system, making itself a new variant of implicit gradient reconstruction methods $[3,11]$, while at the same time we still use the conventional explicit gradient reconstruction approach for all the internal cells. It is worth noted that in Ref.[11] the implicit method is derived from a discretization of a hyperbolic diffusion system, resulting second-order accurate solutions even for highly-curved high-aspect-ratio grids. We expect that calculating the boundary solutions in an implicit manner may also result more accurate and robust approximation of the cell-centered FVM.

The remainder of this article is organized as follows. In the next section, basic formulas of FVM are introduced, and then in section 3 several conventional gradient reconstruction methods are briefly introduced. Specifically, in section 4.1, the vertex-based WLSQ (VWLSQ) method is detailed and extended to 3D, and then in section 4 the present iterative scheme is introduced based on the VWLSQ method. Various numerical test cases are given in sections 5 and 6 to show the advantage of the present method. Eventually, concluding remarks are given in the last section.

\section{Governing equations and the discretization}

In this work, numerical methods for simulating inviscid flows are specifically investigated, and thus the threedimensional Euler equations are applied as the governing equations. The spatial discretization is performed by using the second-order cell-centered finite volume method (FVM). The integral form of the equations is written as:

$$
\frac{\partial}{\partial t} \int_{\Omega} \mathbf{Q} d \Omega+\int_{\partial \Omega} \mathbf{F}(\mathbf{Q}) \cdot \mathbf{n} d s=\mathbf{0},
$$

where $\mathbf{Q}$ is the conservative variables, $\mathbf{F}$ is the convective fluxes, $\Omega$ is the control volume, $\partial \Omega$ is the surface of the control volume, and $\mathbf{n}=\left(n_{x}, n_{y}, n_{z}\right)^{\mathrm{T}}$ is the outward normal vector of the surface.

The conservative variables and convective fluxes of three-dimensional flows are

$$
\mathbf{Q}=\rho\left(\begin{array}{c}
1 \\
u \\
v \\
w \\
e
\end{array}\right), \quad \mathbf{F} \cdot \mathbf{n}=(\mathbf{v} \cdot \mathbf{n})\left(\begin{array}{c}
\rho \\
\rho u \\
\rho v \\
\rho w \\
\rho e+p
\end{array}\right)+p\left(\begin{array}{c}
0 \\
n_{x} \\
n_{y} \\
n_{z} \\
0
\end{array}\right),
$$

where $\rho, p, e$, respectively, represent the density, static pressure and specific total energy per unit volume, and $\mathbf{v}=$ $(u, v, w)^{\mathrm{T}}$ is the velocity vector. The specific total energy is given by

$$
e=\frac{1}{2}\left(u^{2}+v^{2}+w^{2}\right)+\frac{p}{\rho(\gamma-1)},
$$

where $\gamma$ denotes the specific heat ratio, which is given as $\gamma=1.4$ for calorically perfect gas used in this work.

We currently assume that cell-centered FVM solutions are defined at the centroid of grid cells. The semi-discrete form of Eq.(1) on an arbitrary polyhedron or polygon cell $i$ is:

$$
\frac{\mathrm{d}}{\mathrm{d} t}(\mathbf{Q} \Omega)_{i}=-\left(\sum_{k=1}^{N_{f}} \mathbf{F}_{k} \cdot \mathbf{n}_{k} S_{k}\right)_{i},
$$


where cell $i$ shares an interface $k$ with a neighbouring cell $j, \mathbf{n}_{k}$ is the unit outward normal vector of the interface, $S_{k}$ is the interface area, and $N_{f}$ is the amount of faces of cell $i$. The numerical flux $\mathbf{F}_{k}$ is calculated using several popular upwind numerical flux schemes [38, 39, 40,41]. Although the performance of numerical flux schemes is essential for the overall performance of FVM [42, 43], it is not specifically discussed in this work for simplicity.

For a second-order scheme, interface variables $\mathbf{q}_{k}$ are extrapolated from cell-center using the gradients $\nabla \mathbf{q}_{i}$ and the distance vectors $\mathbf{r}_{i k}$ :

$$
\mathbf{q}_{k}=\mathbf{q}_{i}+\phi_{i} \nabla \mathbf{q}_{i} \cdot \Delta \mathbf{r}_{i k},
$$

where $\Delta \mathbf{r}_{i k}=\mathbf{r}_{k}-\mathbf{r}_{i}$, and $\phi_{i} \in[0,1]$ is a slope limiter which is usually employed to suppress oscillations at captured discontinuities [44]. Moreover, the spatial reconstruction procedure in boundary cells and faces is to be specifically discussed in the following sections. It should be noted that in this work we use the primitive variables to perform the spatial reconstruction, and then the conservative variables are calculated based on the reconstructed primitive variables.

Eventually, after performing the spatial solution schemes, the semi-discretized equations (4) are solved by using the implicit lower-upper symmetric-Gauss-Seidel (LU-SGS) scheme [45] for steady cases, and the dual-time stepping [46] for unsteady cases.

\section{Typical methods}

The purpose of the present work is to improve the near-boundary approximation of the second-order FVM. Therefore, classical spatial reconstruction methods $[20,47]$ are introduced at first.

\subsection{GG method}

As the name implies, the Green-Gauss reconstruction method is based on the Gauss divergence theorem. The gradient of a scalar variable $\varphi$ in an arbitrary closed volume can be obtained by

$$
\int_{V} \nabla \varphi d V=\oint_{\partial V} \varphi \mathbf{n} d S,
$$

where $V$ and $\partial V$ are the closed volume and its surface respectively, and $\mathbf{n}$ is the unit outward normal to the surface.

For second-order schemes with linear assumption, the gradient within the element is a constant vector. Therefore, Eq.(6) can be discretized as

$$
\nabla \varphi=\frac{1}{V} \sum_{k=1}^{N_{f}} \bar{\varphi}_{k} \mathbf{n}_{k} S_{k},
$$

where $S_{k}$ is the area of interface $k$, and $\bar{\varphi}_{k}$ is the average of the scalar value over the interface, which is equal to the face-center value in a second-order scheme. It can be found in Eq.(7) that the accuracy of the cell gradient is determined by the accuracy of the face-center value, which needs to be exact for linear functions. Two typical approaches calculating the face-center value are reviewed below.

\subsubsection{Cell averaging (CA) approximation}

The cell averaging method [20] is a basic and simple approach to approximate the face-center value, which is implemented by calculating the arithmetic average of the cell-center values on both sides of the interface (see Fig.1(a)):

$$
\bar{\varphi}_{k}=\frac{\varphi_{l}+\varphi_{r}}{2},
$$

where the subscripts $l$ and $r$ respectively represent the cells on the left and right sides of the interface $k$.

After attaining the face-center variables, Eq.(7) can be calculated, and thus the cell gradient can be given. This approximate approach is noted as CAGG in this paper. This method is feasible for rectangular grids. Whereas, if the grid cells are distorted, directly averaging the cell-center solutions usually results significant errors, because the face center is not longer located at the midpoint of the centroid of the two cells across the face, losing the linear exactness. Accuracy can be recovered by evaluating the face-center value by the average of two solutions linearly interpolated from two cells using the gradients, which are unknowns and can be provided by an implicit method, e.g. the implicit Green-Gauss gradient reconstruction [11]. 


\subsubsection{Node averaging (NA) approximation}

Another more popular mean to attain face-center variables is the node (vertex) averaging method, which is implemented by calculating the arithmetic average of the values at all nodes of a face, i.e.

$$
\bar{\varphi}_{k}=\frac{1}{N_{v}} \sum_{a=1}^{N_{v}} \varphi_{a},
$$

where $\varphi_{a}$ and $N_{v}$ respectively represent the nodal variables and the amount of vertexes of the face. The nodal variables are obtained by the weighted average of cell-center values around the node. The schematic is shown in Fig.1(b) and the formula is

$$
\varphi_{a}=\frac{\sum_{i=1}^{N_{c}} \omega_{i} \varphi_{i}}{\sum_{i=1}^{N_{c}} \omega_{i}},
$$

where $\omega_{i}$ and $N_{c}$ are the weight of a surrounding cell and the number of cells adjacent to the node, respectively. This approximate approach of the Green-Gauss reconstruction method is referred to herein as NAGG. It should be noted that this weighted average cannot guarantee providing exact solutions for linear functions on general unstructured meshes.

Obviously, the weights in Eq.(10) will have a direct impact on the accuracy of the gradient reconstructed by the NAGG method. Several different weighting methods are available, and the inverse distance weighting (IDW) method [15] is robust and efficient one. The IDW is

$$
\omega_{i}^{\mathrm{IDW}}=\frac{1}{L_{a i}},
$$

where $L_{a i}$ is the distance between a node $a$ and the center of a surrounding cell $i$. Another method obtaining weights which satisfy the pseudo-Laplacian relationship was introduced in Refs. [14, 16]. The so-called pseudo-Laplacian weighting (PLW) procedure solves a constrained minimization problem, and has been shown to produce accurate linear reconstructions on regular grids. However, the weights may be negative on stretched or distorted meshes, which means that computational stability can be damaged on common grid layouts [24,25]. Therefore, it is necessary to clip the weights to ensure computational stability, but this will inevitably deteriorate the accuracy of spatial reconstructions $[14,48]$. An essentially similar method is calculating the nodal solutions by using WLSQ methods [19, 49], but still clipping procedure is a necessity for these methods [49].

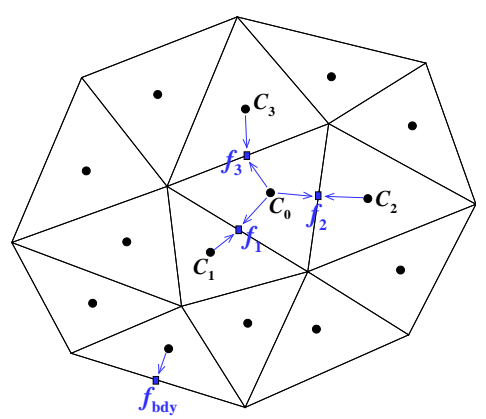

(a) CAGG

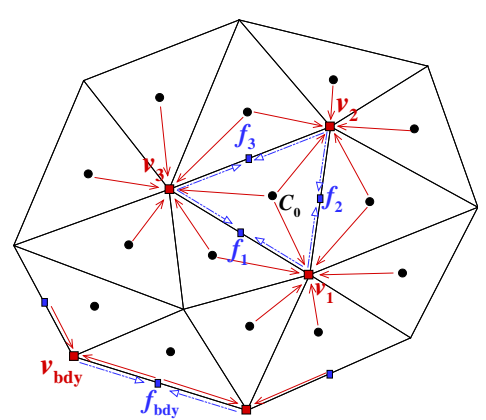

(b) NAGG

Fig. 1. The stencils of the Green-Gauss method.

\subsection{WLSQ method}

The WLSQ method is a linear approximation of the values at a given position by solving a minimization function on the weighted residuals, and in the second-order FVM the WLSQ methods are usually used for calculating the cell center gradients. The stencils used in the WLSQ methods for gradient reconstructions are shown in Fig.2, where the gradient at the cell 0 is to be calculated and cell $i(i=1,2, \cdots)$ is the common-face neighbor of cell 0 . For a 
boundary cell $c_{\text {bdy }}$, two approaches can be used to provide stencils. The first approach is to add the ghost cell(s) to the stencil of the boundary cell, as shown in Fig.2(a), and thus the corresponding WLSQ method is denoted as WLSQ-GC (Ghost-Cell). Another approach is to use all neighbor cells that share at least one node with the boundary cell, as shown in Fig.2(b), and thus the method is denoted as WLSQ-IC (Internal-Cell). For simplicity, in this work, the WLSQ-GC method and WLSQ-IC method use different stencils only for the boundary cells, but use the same stencil for the internal cells. It should be noted that, if the stencil is regular or symmetry, WLSQ methods are able to provide second-order accurate solutions. On irregular grids, WLSQ gradients will be first-order accurate since the algorithm is designed to be exact for linear functions, which is sufficient for giving second-order accuracy in the solution variables. However, WLSQ-GC methods can lose linear exactness if it relies on the solutions in ghost cells, which are not usually calculated exactly for linear functions.

For instance, in order to solve the Euler equations, the geometry property of ghost cells can be obtained by mirroring the cell $c_{\text {bdy }}$ with respect to the boundary surface $f_{\text {bdy }}$. Then we have

$$
\mathbf{r}_{\mathrm{c}_{\text {ghost }}}=\mathbf{r}_{\mathrm{c}_{\mathrm{bdy}}}-2\left[\left(\mathbf{r}_{\mathrm{c}_{\mathrm{bdy}}}-\mathbf{r}_{\mathrm{f}_{\mathrm{bdy}}}\right) \cdot \mathbf{n}_{\mathrm{f}_{\mathrm{bdy}}}\right] \cdot \mathbf{n}_{\mathrm{f}_{\mathrm{bdy}}},
$$

where $\mathbf{r}_{(\cdot)}=\left(x_{(\cdot)}, y_{(\cdot)}, z_{(\cdot)}\right)$ is the coordinate of the corresponding location and $\mathbf{n}_{\mathrm{f}_{\mathrm{bdy}}}$ is the unit normal vector of the boundary surface. The solutions in ghost cells are given by the first-order extrapolation, and boundary conditions are taken into account to assign the density, pressure and velocity, i.e.

$$
\rho_{\mathrm{c}_{\text {ghost }}}=\rho_{\mathrm{c}_{\mathrm{bdy}}}, \quad p_{\mathrm{c}_{\mathrm{ghhost}}}=p_{\mathrm{c}_{\mathrm{bdy}}}, \quad \text { and } \quad \mathbf{V}_{\mathrm{c}_{\mathrm{ghost}}}=\mathbf{V}_{\mathrm{c}_{\mathrm{bdy}}}-2\left(\mathbf{V}_{\mathrm{c}_{\mathrm{bdy}}} \cdot \mathbf{n}_{\mathrm{f}_{\mathrm{bdy}}}\right) \cdot \mathbf{n}_{\mathrm{f}_{\mathrm{bdy}}},
$$

where $\mathbf{V}$ is the vector of velocity. For simplicity, more boundary conditions used for ghost cells are not further discussed here, since they are commonly used.

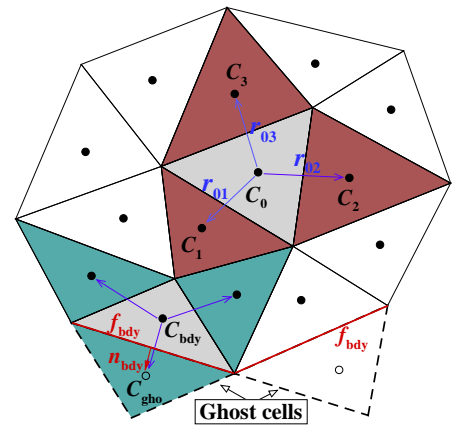

(a) WLSQ-GC

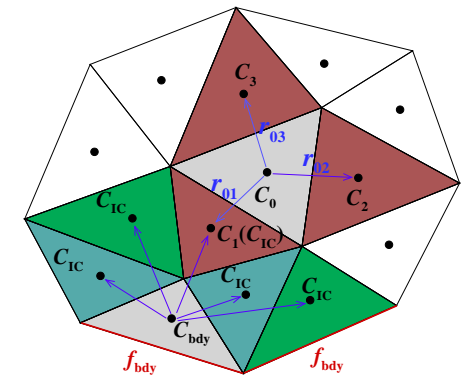

(b) WLSQ-IC

Fig. 2. The stencils of the WLSQ method.

The Taylor series expansion of the variable $\varphi_{i}$ at the $i^{\text {th }}$ stencil (cell center) can be written as (only the first derivative term needs to be considered for second-order schemes):

$$
\varphi_{i}=\varphi_{0}+\nabla \varphi_{0} \cdot \Delta \mathbf{r}_{0 i}+\text { h.o.t. } \approx \varphi_{0}+\frac{\partial \varphi_{0}}{\partial x} \Delta x_{0 i}+\frac{\partial \varphi_{0}}{\partial y} \Delta y_{0 i}+\frac{\partial \varphi_{0}}{\partial z} \Delta z_{0 i}
$$

where $\Delta \mathbf{r}_{0 i}=\left(\Delta x_{0 i}, \Delta y_{0 i}, \Delta z_{0 i}\right)^{\mathrm{T}}$ is the distance vector from the center of cell 0 to the center of a surrounding cell $i$. In Eq.(14), only the derivative $\nabla \varphi_{0}$ is unknown. The function of weighted sum of squared residuals is given as

$$
f\left(\omega_{i}, \varphi_{i}, \varphi_{0}, \frac{\partial \varphi_{0}}{\partial x}, \frac{\partial \varphi_{0}}{\partial y}, \frac{\partial \varphi_{0}}{\partial z}\right)=\sum_{i=1}^{N_{c}} \omega_{i}\left(\varphi_{i}-\varphi_{0}-\frac{\partial \varphi_{0}}{\partial x} \Delta x_{0 i}-\frac{\partial \varphi_{0}}{\partial y} \Delta y_{0 i}-\frac{\partial \varphi_{0}}{\partial z} \Delta z_{0 i}\right)^{2}
$$

where $\omega_{i}$ represents the weight of each neighbor cell $i$, and $N_{c}$ is the number of neighbors sharing an interface with 
cell 0. The function $f\left(\omega_{i}, \varphi_{i}, \varphi_{0}, \frac{\partial \varphi_{0}}{\partial x}, \frac{\partial \varphi_{0}}{\partial y}, \frac{\partial \varphi_{0}}{\partial z}\right)$ is required to be minimized, then we have:

$$
\left\{\begin{array}{l}
\frac{\partial f}{\partial \varphi_{0, x}}=-\sum_{i=1}^{N_{c}} 2 \omega_{i}\left(\Delta \varphi_{0 i}-\varphi_{0, x} \Delta x_{0 i}-\varphi_{0, y} \Delta y_{0 i}-\varphi_{0, z} \Delta z_{0 i}\right) \Delta x_{0 i}=0 \\
\frac{\partial f}{\partial \varphi_{0, y}}=-\sum_{i=1}^{N_{c}} 2 \omega_{i}\left(\Delta \varphi_{0 i}-\varphi_{0, x} \Delta x_{0 i}-\varphi_{0, y} \Delta y_{0 i}-\varphi_{0, z} \Delta z_{0 i}\right) \Delta y_{0 i}=0 \\
\frac{\partial f}{\partial \varphi_{0, z}}=-\sum_{i=1}^{N_{c}} 2 \omega_{i}\left(\Delta \varphi_{0 i}-\varphi_{0, x} \Delta x_{0 i}-\varphi_{0, y} \Delta y_{0 i}-\varphi_{0, z} \Delta z_{0 i}\right) \Delta z_{0 i}=0
\end{array}\right.
$$

where $\varphi_{0,(\cdot)}=\frac{\partial \varphi_{0}}{\partial(\cdot)}$, and $\Delta \varphi_{0 i}=\varphi_{i}-\varphi_{0}$. It is shown that Eq.(16) is a linear system which can be written as

$$
\mathbf{A}_{0} \mathbf{X}_{0}=\mathbf{B}_{0}
$$

with

$$
\begin{gathered}
\mathbf{A}_{0}=\left[\begin{array}{lll}
\sum_{i=1}^{N_{c}} \omega_{i} \Delta x_{0 i}^{2} & \sum_{i=1}^{N_{c}} \omega_{i} \Delta x_{0 i} \Delta y_{0 i} & \sum_{i=1}^{N_{c}} \omega_{i} \Delta x_{0 i} \Delta z_{0 i} \\
\sum_{i=1}^{N_{c}} \omega_{i} \Delta x_{0 i} \Delta y_{0 i} & \sum_{i=1}^{N_{c}} \omega_{i} \Delta y_{0 i}^{2} & \sum_{i=1}^{N_{c}} \omega_{i} \Delta y_{0 i} \Delta z_{0 i} \\
\sum_{i=1}^{N_{c}} \omega_{i} \Delta x_{0 i} \Delta z_{0 i} & \sum_{i=1}^{N_{c}} \omega_{i} \Delta y_{0 i} \Delta z_{0 i} & \sum_{i=1}^{N_{c}} \omega_{i} \Delta z_{0 i}^{2}
\end{array}\right] \\
\mathbf{B}_{0}=\left[\begin{array}{l}
\sum_{i=1}^{N_{c}} \omega_{i} \Delta \varphi_{0 i} \Delta x_{0 i} \\
\sum_{i=1}^{N_{c}} \omega_{i} \Delta \varphi_{0 i} \Delta y_{0 i} \\
\sum_{i=1}^{N_{c}} \omega_{i} \Delta \varphi_{0 i} \Delta z_{0 i}
\end{array}\right], \quad \mathbf{X}_{0}=\left[\begin{array}{l}
\frac{\partial \varphi_{0}}{\partial x} \\
\frac{\partial \varphi_{0}}{\partial y} \\
\frac{\partial \varphi_{0}}{\partial z}
\end{array}\right]
\end{gathered}
$$

In Eqs.(15)-(18), the weight $\omega_{i}$ can also be determined by the inverse distance and written in an universal form as

$$
\omega_{i}=\frac{1}{L_{0 i}^{n}},
$$

where $L_{0 i}$ is the distance from cell $i$ to cell 0 , and $n=0,1,2 \cdots$. Obviously, $n=0$ means an unweighted process. For the convenience of discussion, the WLSQ methods using different weight functions are denoted as WLSQ(n).

\section{The iterative near-boundary reconstruction strategy}

The accuracy of gradients in internal cells with using different methods has been extensively discussed in numerous literatures. In general, on regular grids, the linear exactness can be achieved by using various methods. However, it is not trivial to guarantee the linear exactness on general mesh types. Moreover, the solutions at boundary face quadrature points (face centroid) are used for calculating the numerical fluxes, and these solutions need to be exact for linear functions, otherwise the linear exactness will be lost as well.

In this section, the VWLSQ-averaged-gradient method (simply denoted as VWLSQ method) [24, 25] is introduced at first, which is also extended to 3D, and then a novel iterative strategy is introduced for improving the near-boundary approximation. Finally, a vectorial limiter is introduced to guarantee the correctness of the present iterative solutions.

\subsection{The VWLSQ method}

The present work is based on the VWLSQ method which was proposed for cell gradient reconstruction in an earlier work [24, 25]. Unlike the cell-based WLSQ methods, which reconstruct cell gradients directly using surrounding stencils, the VWLSQ method reconstructs the gradients at the nodes (vertexes) at first, and then the cell or face gradient can be calculated by averaging the nodal gradients. The stencils used in the VWLSQ method is shown in Fig.3, and obviously, for the boundary vertexes, the ghost cells can also be used as supplementary stencils. 


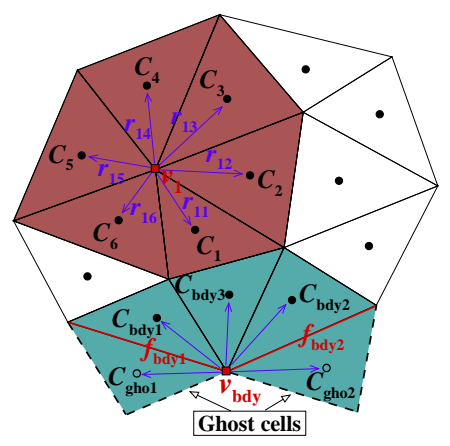

Fig. 3. VWLSQ stencils.

By considering the Taylor series expansion, the cell-center variable $\varphi_{i}$ can be written as the following form similar to Eq.(14):

$$
\varphi_{i}=\varphi_{a}+\nabla \varphi_{a} \cdot \Delta \mathbf{r}_{a i}+\text { h.o.t. } \approx \varphi_{a}+\frac{\partial \varphi_{a}}{\partial x} \Delta x_{a i}+\frac{\partial \varphi_{a}}{\partial y} \Delta y_{a i}+\frac{\partial \varphi_{a}}{\partial z} \Delta z_{a i}
$$

where $\Delta \mathbf{r}_{a i}=\left(\Delta x_{a i}, \Delta y_{a i}, \Delta z_{a i}\right)^{\mathrm{T}}$ is the distance vector from the vertex $a$ to the center of a surrounding cell $i$. It should be noted that both the nodal value $\varphi_{a}$ and its gradient $\nabla \varphi_{a}$ are unknown here. In order to calculate these unknowns, the idea of least squares is applied. The function of the weighted sum of squared residuals is given as

$$
f\left(\omega_{i}, \varphi_{i}, \varphi_{a}, \frac{\partial \varphi_{a}}{\partial x}, \frac{\partial \varphi_{a}}{\partial y}, \frac{\partial \varphi_{a}}{\partial z}\right)=\sum_{i=1}^{N_{c}} \omega_{i}\left(\varphi_{i}-\varphi_{a}-\frac{\partial \varphi_{a}}{\partial x} \Delta x_{a i}-\frac{\partial \varphi_{a}}{\partial y} \Delta y_{a i}-\frac{\partial \varphi_{a}}{\partial z} \Delta z_{a i}\right)^{2}
$$

where $\omega_{i}$ represents the weight of a surrounding cell $i$, and $N_{c}$ is the number of adjacent cells surrounding the vertex $a$. The function $f\left(\omega_{i}, \varphi_{i}, \varphi_{a}, \frac{\partial \varphi_{a}}{\partial x}, \frac{\partial \varphi_{a}}{\partial y}, \frac{\partial \varphi_{a}}{\partial z}\right)$ is to be minimized, and then we have:

$$
\left\{\begin{aligned}
\frac{\partial f}{\partial \varphi_{a}} & =-\sum_{i=1}^{N_{c}} 2 \omega_{i}\left(\varphi_{i}-\varphi_{a}-\varphi_{a, x} \Delta x_{a i}-\varphi_{a, y} \Delta y_{a i}-\varphi_{a, z} \Delta z_{a i}\right)=0 \\
\frac{\partial f}{\partial \varphi_{a, x}} & =-\sum_{i=1}^{N_{c}} 2 \omega_{i}\left(\varphi_{i}-\varphi_{a}-\varphi_{a, x} \Delta x_{a i}-\varphi_{a, y} \Delta y_{a i}-\varphi_{a, z} \Delta z_{a i}\right) \Delta x_{a i}=0 \\
\frac{\partial f}{\partial \varphi_{a, y}} & =-\sum_{i=1}^{N_{c}} 2 \omega_{i}\left(\varphi_{i}-\varphi_{a}-\varphi_{a, x} \Delta x_{a i}-\varphi_{a, y} \Delta y_{a i}-\varphi_{a, z} \Delta z_{a i}\right) \Delta y_{a i}=0 \\
\frac{\partial f}{\partial \varphi_{a, z}} & =-\sum_{i=1}^{N_{c}} 2 \omega_{i}\left(\varphi_{i}-\varphi_{a}-\varphi_{a, x} \Delta x_{a i}-\varphi_{a, y} \Delta y_{a i}-\varphi_{a, z} \Delta z_{a i}\right) \Delta z_{a i}=0
\end{aligned}\right.
$$

where $\varphi_{a,(\cdot)}=\frac{\partial \varphi_{a}}{\partial(\cdot)}$. It is shown that Eq.(22) leads to a linear system that has one more dimension compared with Eq.(16), and the linear system can be written as:

$$
\mathbf{A}_{a} \mathbf{X}_{a}=\mathbf{B}_{a}
$$


with

$$
\begin{array}{rlll}
\mathbf{A}_{a}=\left[\begin{array}{llll}
\sum_{i=1}^{N_{c}} \omega_{i} & \sum_{i=1}^{N_{c}} \omega_{i} \Delta x_{a i} & \sum_{i=1}^{N_{c}} \omega_{i} \Delta y_{a i} & \sum_{i=1}^{N_{c}} \omega_{i} \Delta z_{a i} \\
\sum_{i=1}^{N_{c}} \omega_{i} \Delta x_{a i} & \sum_{i=1}^{N_{c}} \omega_{i} \Delta x_{a i}^{2} & \sum_{i=1}^{N_{c}} \omega_{i} \Delta x_{a i} \Delta y_{a i} & \sum_{i=1}^{N_{c}} \omega_{i} \Delta x_{a i} \Delta z_{a i} \\
\sum_{i=1}^{N_{c}} \omega_{i} \Delta y_{a i} & \sum_{i=1}^{N_{c}} \omega_{i} \Delta x_{a i} \Delta y_{a i} & \sum_{i=1}^{N_{c}} \omega_{i} \Delta y_{a i}^{2} & \sum_{i=1}^{N_{c}} \omega_{i} \Delta y_{a i} \Delta z_{a i} \\
\sum_{i=1}^{N_{c}} \omega_{i} \Delta z_{a i} & \sum_{i=1}^{N_{c}} \omega_{i} \Delta x_{a i} \Delta z_{a i} & \sum_{i=1}^{N_{c}} \omega_{i} \Delta y_{a i} \Delta z_{a i} & \sum_{i=1}^{N_{c}} \omega_{i} \Delta z_{a i}^{2}
\end{array}\right], \\
\mathbf{B}_{a}=\left[\begin{array}{c}
\sum_{i=1}^{N_{c}} \omega_{i} \varphi_{i} \\
\sum_{i=1}^{N_{c}} \omega_{i} \varphi_{i} \Delta x_{a i} \\
\sum_{i=1}^{N_{c}} \omega_{i} \varphi_{i} \Delta y_{a i} \\
\sum_{i=1}^{N_{c}} \omega_{i} \varphi_{i} \Delta z_{a i}
\end{array}\right], \quad \mathbf{X}_{a}=\left[\begin{array}{c}
\varphi_{a} \\
\frac{\partial \varphi_{a}}{\partial x} \\
\frac{\partial \varphi_{a}}{\partial y} \\
\frac{\partial \varphi_{a}}{\partial z}
\end{array}\right]
\end{array}
$$

In Eqs.(21)-(24), the weight is also determined by the inverse distance:

$$
\omega_{i}=\frac{1}{L_{a i}^{n}},
$$

where $L_{a i}$ is the distance from node $a$ to cell $i$, and $n=0,1,2, \cdots$.

It should be noted that, in this work, the ghost cells, e.g. in Fig.3, are replaced by the boundary surfaces as the supplementary stencils, as shown in Fig.4. For this stencil, Eq.(20) will firstly be replaced by

$$
\varphi_{\mathrm{bdy}} \approx \varphi_{a}+\nabla \varphi_{a} \cdot \Delta \mathbf{r}_{a, \mathrm{bdy}}
$$

where $\varphi_{\text {bdy }}$ and $\Delta \mathbf{r}_{a \text {,bdy }}$ are respectively the values of the variables at the boundary surface center and the distance vector from vertex $a$ to $f_{\text {bdy }}$. This basic boundary treatment helps to implement the following iterative strategy.

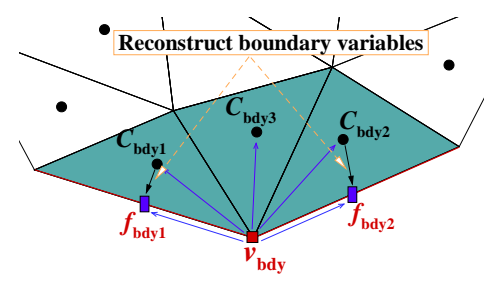

Fig. 4. The stencil of a boundary vertex for the present method.

It should be noted that, as shown in Eq.(20), the amount of unknowns per vertex is equal to dim +1 (dim is the spatial dimension of the mesh), which are more than those in a WLSQ cell-gradient reconstruction, so at least $\operatorname{dim}+1$ equations are required to determine these unknowns. Fortunately, this requirement is always satisfied on arbitrary polygon or polyhedra meshes. Taking a 2D mesh as an example, each internal vertex is adjacent to at least three lines, which means that at least three cells are sharing the vertex, so that at least three linear equations can be established. A boundary vertex is adjacent to at least one internal cell and two ghost cells or boundary surfaces, which will also provide at least three linear equations. Obviously, there is a similar conclusion for 3D cases, that is, the number of equations for an internal vertex or a boundary vertex is not less than four, which means the linear equations have a unique solution.

So far, the solutions at vertexes and their gradients are obtained. These nodal information can be used for the cell 
gradient or interface gradient reconstruction [24, 25], but in this work only the cell gradients are calculated as

$$
\nabla \varphi_{c}=\frac{\sum_{a=1}^{N_{v}} \omega_{a} \nabla \varphi_{a}}{\sum_{a=1}^{N_{v}} \omega_{a}},
$$

where $N_{v}$ is the amount of vertexes adjacent to the cell, and $\omega_{a}$ represents the weight of vertex $a$, which has a similar form of Eq.(25):

$$
\omega_{a}=\frac{1}{L_{a i}^{m}},
$$

where $L_{a i}$ is the distance between the vertex $a$ and the center of a surrounding cell $i$. In this paper, depending on the weights defined in Eqs.(25) and (28), the gradient reconstruction method described in this section is uniformly written as VWLSQ $(n-m)$. It should be noted that in the original VWLSQ method [24, 25] this averaging procedure is not weighted.

The VWLSQ method requires solving a linear system at each vertex, which is a $(\operatorname{dim}+1) \times(\operatorname{dim}+1)$ symmetric matrix, and this system can be solved efficiently. Moreover, compared with the cell-based WLSQ method, the VWLSQ method has advantages in terms of storage and computational cost [12, 24, 25]. For different types of meshes, the amounts of vertexes, cells, and faces are respectively shown in Table 1, excluding the influence of boundaries. It can be seen that an inequality $N_{v} \leq N_{c}<N_{f}$ is always valid for any type of mesh, and $N_{v}$ is equal to $N_{c}$ only on quadrilateral and hexahedral meshes. Therefore, computing the least squares problem at the vertexes is more cost-effective compared with computing at cells or at surfaces.

Table 1. The amount of vertexes, cells, and faces on different grid types.

\begin{tabular}{ccccccc}
\hline & Regular Triangle & Quadrilateral & Regular Tetrahedron & Pyramid & Prism & Hexahedron \\
\hline$N_{v}$ (Vertex) & $N_{\text {Tri }}$ & $N_{\text {Qua }}$ & $N_{\text {Tet }}$ & $N_{\text {Pyr }}$ & $N_{\text {Pri }}$ & $N_{\text {Hex }}$ \\
$N_{c}$ (Cell) & $2 N_{\text {Tri }}$ & $N_{\text {Qua }}$ & $6 N_{\text {Tet }}$ & $6 N_{\text {Pyr }}$ & $2 N_{\text {Pri }}$ & $N_{\text {Hex }}$ \\
$N_{f}$ (Face) & $3 N_{\text {Tri }}$ & $2 N_{\text {Qua }}$ & $12 N_{\text {Tet }}$ & $15 N_{\text {Pyr }}$ & $5 N_{\text {Pri }}$ & $3 N_{\text {Hex }}$ \\
\hline
\end{tabular}

\subsection{An iterative strategy}

The VWLSQ method first needs to calculate the gradients at vertexes, but $\varphi_{\text {bdy }}$ in Eq.(26) also needs to be calculated. Apparently, using the first-order extrapolation approach mentioned above to calculate $\varphi_{\text {bdy }}$ cannot recover the linear exactness, and the VWLSQ procedure will then cause error in the reconstructed gradients at boundary vertexes. Therefore, we propose a new iterative strategy, in which boundary conditions are taken into account, to calculate the gradients in the boundary cells and the solutions at the boundary faces altogether. The iteration procedure is as follows:

(1) Update the reference solutions at the centers of all boundary surfaces:

$$
\tilde{\varphi}_{\text {bdy }}^{n}=\varphi_{c}+\nabla \varphi_{c}^{n} \cdot \Delta \mathbf{r}_{c, \text { bdy }}
$$

For the first iteration step $\nabla \varphi_{c}^{0}$ is equal to zero.

(2) Correct $\tilde{\varphi}_{\text {bdy }}^{n}$ according to the boundary constraints to obtain a physical boundary value $\varphi_{\text {bdy }}^{n}$. For example, for the slip wall BC, the component of the velocity vector in the normal direction of the boundary surface is equal to zero to satisfy the non-penetration constraint.

(3) Calculate the gradients $\nabla \varphi_{v}^{n}$ at the boundary vertexes according to Eqs.(20)- (26).

(4) Update the gradients $\tilde{\nabla} \varphi_{c}^{n+1}$ in the boundary cells according to Eqs.(27) and (28).

(5) Limit the gradient $\tilde{\nabla} \varphi_{c}^{n+1}$ to avoid iterative oscillations:

$$
\nabla \varphi_{c}^{n+1}=\mathcal{L}\left(\tilde{\nabla} \varphi_{c}^{n+1}\right)
$$

where $\mathcal{L}$ is a limiting process that will be described in detail in section 4.3.

(6) Repeat the above steps (1)-(2) to update the boundary values $\varphi_{\text {bdy }}^{n+1}$. 
(7) Calculate the relative error of the boundary values:

$$
e r r_{\text {iter }}=\left(\left\|\frac{\varphi_{\text {bdy }}^{n+1}-\varphi_{\text {bdy }}^{n}}{\varphi_{\text {bdy }}^{n}}\right\|_{\infty}\right)_{\partial \Omega},
$$

where $\|\cdot\|$ is the norm computed over the entire computational domain.

(a) If $e r r_{\text {iter }} \leq \varepsilon$ or $n_{\text {iter }}=N_{\text {iter }}^{\max }$ : Stop.

(b) Otherwise: repeat steps (3)-(7).

where $n_{\text {iter }}$ is the current step of iterations, $N_{\mathrm{iter}}^{\max }$ is the maximum limit of iteration steps allowed, and $\varepsilon$ is a small number $0<\varepsilon \ll 1$.

Eventually, with using the VWLSQ method and this iterative strategy as the building blocks, the present spatial reconstruction method is denoted as VWLSQ-ITER method. It should be noted that, since the gradient at a internal node depends only on its surrounding cells whose values are not changed by the iteration procedure, it will not change during the iterations, and because the boundary nodes are independent to internal nodes, the error of the iteration procedure will not affect the interior in one temporal step.

Although the accuracy of the gradients in boundary cells can be effectively improved by the iterative operation, it is not desirable to introduce too much computational cost in this process. Fortunately, the iterative strategy is only activated for boundary cells and faces, and thus the computational effort is insignificant because the number of boundary nodes is very small compared with the number of internal nodes. The extra cost of the iteration computation is

$$
\operatorname{cost}_{\mathrm{iter}}=\left(\frac{N_{b v}}{N_{i v}+N_{b v}}\right) \times \operatorname{cost} t_{\mathrm{all}} \times n_{\mathrm{iter}},
$$

where $N_{b v}, N_{i v}$ and cost $t_{\text {all }}$ are the amount of boundary nodes, the amount of internal nodes and the cost of calculating the gradients at all nodes, respectively. As can be seen in Eqs.(24), the matrix $\mathbf{A}$ is fixed during the iteration, and only vector $\mathbf{B}$ needs to be updated. Therefore, if the mesh is adaptively refined, the computational effort of the present method is still only proportional to the amount of boundary vertexes.

\subsection{A vectorial limiter}

Ideally, it is undesirable to use limiters for spatial reconstructions, to avoid potential accuracy deterioration. However, on highly distorted/curved grids, or in complex flow fields including shock waves, the accuracy and positivity of gradient reconstruction results can be doubtful. In this work, we have not intention to provide a theoretical proof of the convergence or stability. Instead, a numerical technique is implemented, to avoid numerical oscillation or violating the geometrical monotonicity which is critical on high-aspect-ratio curved grids [23].

It is straightforward to implement slope limiters $[20,50]$ for the purpose mentioned above. However, conventional slope limiters are in fact scalar limiters which means that there is a limiting value $\phi$ acting on the gradient (which is a vector). Specifically, due to boundary effects, the flows in boundary cells will exhibit anisotropic properties, and thus a scalar limiter is too dissipative. Here, a vectorial limiter $[27,28,51]$ is extended to three-dimension and activated in each step of the iterative spatial reconstruction, avoiding potential oscillation and guaranteeing the geometrical monotonicity [23]. However, applying this limiter will probably damage the accuracy of spatial reconstructions, and this shortcoming is accepted in this work to ensure the robustness of the present iterative strategy.

The solution $\varphi$ in cell $c$ is linearly reconstructed as:

$$
\varphi(x, y, z)=\varphi_{c}+\phi_{x} \frac{\partial \varphi_{c}}{\partial x}\left(x-x_{c}\right)+\phi_{y} \frac{\partial \varphi_{c}}{\partial y}\left(y-y_{c}\right)+\phi_{z} \frac{\partial \varphi_{c}}{\partial z}\left(z-z_{c}\right),
$$

where $\phi_{x}, \phi_{y}, \phi_{z} \in[0,1]$. The maximum/minimum principle is used to ensure monotonicity, if certain criteria is triggered. The values reconstructed to all non-boundary surface centers of the cell $c$ will be bounded within

$$
\varphi_{\text {lower }} \leq \varphi_{c}+\left[\begin{array}{c}
\left(x_{k}-x_{c}\right) \frac{\partial \varphi_{c}}{\partial x} \\
\left(y_{k}-y_{c}\right) \frac{\partial \varphi_{c}}{\partial y} \\
\left(z_{k}-z_{c}\right) \frac{\partial \varphi_{c}}{\partial z}
\end{array}\right] \cdot\left[\begin{array}{c}
\phi_{x} \\
\phi_{y} \\
\phi_{z}
\end{array}\right] \leq \varphi_{\text {upper }}, \quad \text { for } k=1, \ldots, N_{c(f)},
$$


where interface $k$ is sharing by cell $c$ and a neighboring cell $c_{i}, N_{c(f)}$ is the amount of neighboring cells sharing a surface with the cell $c$, and $\varphi_{\text {lower }}$ and $\varphi_{\text {upper }}$ respectively represent the minimum and maximum values of the solution in cell $c$ and surrounding stencils. Here, $\varphi_{\text {lower }}$ and $\varphi_{\text {upper }}$ can be expressed in the following forms:

$$
\begin{gathered}
\text { Standard stencil : }\left\{\begin{array}{l}
\varphi_{\text {lower }}=\min \left(\varphi_{c}, \varphi_{c_{1}}, \ldots, \varphi_{c_{N_{c}(f)}}\right) \\
\varphi_{\text {upper }}=\max \left(\varphi_{c}, \varphi_{c_{1}}, \ldots, \varphi_{c_{N_{c}(f)}}\right)
\end{array},\right. \\
\text { Extended stencil : }\left\{\begin{array}{l}
\varphi_{\text {lower }}=\min \left(\varphi_{c}, \varphi_{c_{1}}, \ldots, \varphi_{c_{N_{c(v)}}}\right) \\
\varphi_{\text {upper }}=\max \left(\varphi_{c}, \varphi_{c_{1}}, \ldots, \varphi_{c_{N_{c}}}\right)
\end{array}\right), \\
\text { Full stencil : }\left\{\begin{array}{l}
\varphi_{\text {lower }}=\min \left(\varphi_{c}, \varphi_{c_{1}}, \ldots, \varphi_{c_{N_{c}(v)}}, \varphi_{v_{1}}, \ldots, \varphi_{v_{N_{v}}}\right) \\
\varphi_{\text {upper }}=\max \left(\varphi_{c}, \varphi_{c_{1}}, \ldots, \varphi_{c_{N_{c}}}, \varphi_{v_{1}}, \ldots, \varphi_{v_{N_{v}}}\right)
\end{array},\right.
\end{gathered}
$$

where $N_{c(v)}$ is the amount of neighbors that share at least one vertex with cell $c$, and $N_{v}$ is the amount of vertexes of cell $c$, as shown in Fig.5. It should be noted that the full stencil requires values at the vertexes, which, fortunately, can be obtained simultaneously in the VWLSQ procedure. Obviously, using the standard stencils is more restrictive. The full stencil relaxes the upper and lower bounds, allowing weak local extrema, and thus it is used in this work.

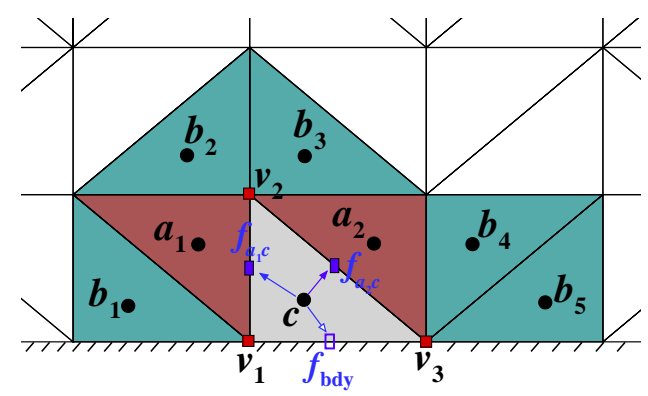

Fig. 5. The maximum/minimum principle stencil of the LP method.

The reconstructed value at the center of a boundary surface is not subject to any constraints except that the pressure and density are required to be positive. Therefore, the inequality form at the boundary is written as

$$
\varphi_{c}+\left[\begin{array}{c}
\left(x_{\mathrm{bdy}}-x_{c}\right) \frac{\partial \varphi_{c}}{\partial x} \\
\left(y_{\mathrm{bdy}}-y_{c}\right) \frac{\partial \varphi_{c}}{\partial y} \\
\left(z_{\mathrm{bdy}}-z_{c}\right) \frac{\partial \varphi_{c}}{\partial z}
\end{array}\right] \cdot\left[\begin{array}{c}
\phi_{x} \\
\phi_{y} \\
\phi_{z}
\end{array}\right]>0,
$$

where $\left(x_{\mathrm{bdy}}, y_{\mathrm{bdy}}, z_{\mathrm{bdy}}\right)$ is the coordinate of the boundary surface center.

In the gradient limiting process, the value of the gradient should be changed as little as possible to reduce the numerical dissipation, while satisfying the monotonic condition. The $L_{1}$-norm of the difference between the limited and unlimited gradients will be used to measure the variation of the gradient. Then the objective function is designed as

$$
\begin{aligned}
\operatorname{minimize} & \left|\frac{\partial \varphi}{\partial x}-\phi_{x} \frac{\partial \varphi}{\partial x}\right|+\left|\frac{\partial \varphi}{\partial y}-\phi_{y} \frac{\partial \varphi}{\partial y}\right|+\left|\frac{\partial \varphi}{\partial z}-\phi_{z} \frac{\partial \varphi}{\partial z}\right| \\
= & \left(1-\phi_{x}\right)\left|\frac{\partial \varphi}{\partial x}\right|+\left(1-\phi_{y}\right)\left|\frac{\partial \varphi}{\partial y}\right|+\left(1-\phi_{z}\right)\left|\frac{\partial \varphi}{\partial z}\right| \\
= & -\phi_{x}\left|\frac{\partial \varphi}{\partial x}\right|-\phi_{y}\left|\frac{\partial \varphi}{\partial y}\right|-\phi_{z}\left|\frac{\partial \varphi}{\partial z}\right|+\left|\frac{\partial \varphi}{\partial x}\right|+\left|\frac{\partial \varphi}{\partial y}\right|+\left|\frac{\partial \varphi}{\partial z}\right|,
\end{aligned}
$$

where $\left|\frac{\partial \varphi}{\partial x}\right|+\left|\frac{\partial \varphi}{\partial y}\right|+\left|\frac{\partial \varphi}{\partial z}\right|$ is a constant term. Obviously, Eq.(37) is a constrained optimization problem that satisfies Eqs.(34)-(36) and $\phi_{x}, \phi_{y}, \phi_{z} \in[0,1]$, which can be written in a general form as

$$
\min _{\phi_{x}, \phi_{y}, \phi_{z}}-\phi_{x}\left|\frac{\partial \varphi}{\partial x}\right|-\phi_{y}\left|\frac{\partial \varphi}{\partial y}\right|-\phi_{z}\left|\frac{\partial \varphi}{\partial z}\right|, \quad \text { subject to } \quad \mathbf{A}_{\mathrm{LP}}\left[\begin{array}{c}
\phi_{x} \\
\phi_{y} \\
\phi_{z}
\end{array}\right] \geq \mathbf{b}_{\mathrm{LP}},
$$


with $\mathbf{A}_{\mathrm{LP}} \in \mathbb{R}^{k \times 3}$ and $\mathbf{b}_{\mathrm{LP}} \in \mathbb{R}^{k}$, where $k$ is the number of constraints. This is a typical linear programming (LP) problem, and the optimal solution $\left(\phi_{x}, \phi_{y}, \phi_{z}\right)$ gives the vectorial limiter. An all-inequality simplex algorithm recommended by Ref.[28] will be used to solve this LP problem, which is more efficient than the standard simplex method for the tiny LPs. It is worth noted that $\left(\phi_{x}, \phi_{y}, \phi_{z}\right)=(0,0,0)$ becomes the starting point for the simplex method because it naturally satisfies the inequality constraint in Eqs.(34)-(36).

Moreover, the present vectorial limiting process is incorporated in the iterative spatial reconstruction, which is only applied for gradients in boundary cells, and thus conventional slope limiters can still be used in Eq.(5).

\subsection{Note: boundary conditions}

In subsection 4.2, the iterative spatial reconstruction procedure is defined, and during the iteration the gradients in boundary cells and the solutions at boundary surfaces are updated altogether. In this subsection, the formulas of calculating boundary solutions is explicitly given. Since the present work focuses on the Euler equations, the subsonic far-field BCs, supersonic inflow/outflow BCs and the slip wall BC are introduced.

(1) Subsonic far-field BCs

The far-field BCs are determined by the Riemann invariants, the boundary variables are given as

$$
\left\{\begin{array}{l}
\rho_{\text {bdy }}=\rho_{\text {ref }}\left(\frac{\rho_{\text {ref }} s_{\text {bdy }}^{2}}{\gamma p_{\text {ref }}}\right)^{\frac{1}{\gamma-1}} \\
u_{\text {bdy }}=u_{\text {ref }}+n_{x}\left(\tilde{V}_{\text {bdy }}-\tilde{V}_{\text {ref }}\right) \\
v_{\text {bdy }}=v_{\text {ref }}+n_{y}\left(\tilde{V}_{\text {bdy }}-\tilde{V}_{\text {ref }}\right) \\
w_{\text {bdy }}=w_{\text {ref }}+n_{z}\left(\tilde{V}_{\text {bdy }}-\tilde{V}_{\text {ref }}\right) \\
p_{\text {bdy }}=s_{\text {bdy }}^{2} \frac{\rho_{\text {bdy }}}{\gamma}
\end{array}\right.
$$

with

$$
\begin{aligned}
& R^{+}=\mathbf{V}_{\mathrm{L}} \cdot \mathbf{n}_{\text {bdy }}+\frac{2}{\gamma-1} \sqrt{\gamma p_{\mathrm{L}} / \rho_{\mathrm{L}}}, \quad R^{-}=\mathbf{V}_{\infty} \cdot \mathbf{n}_{\mathrm{bdy}}+\frac{2}{\gamma-1} \sqrt{\gamma p_{\infty} / \rho_{\infty}}, \\
& \tilde{V}_{\mathrm{bdy}}=\frac{1}{2}\left(R^{+}+R^{-}\right), \quad \tilde{V}_{\mathrm{ref}}=\mathbf{V}_{\text {ref }} \cdot \mathbf{n}_{\mathrm{bdy}}, \quad s_{\mathrm{bdy}}=\frac{\gamma-1}{4}\left(R^{+}-R^{-}\right),
\end{aligned}
$$

where subscripts $\mathrm{L}$ and $\infty$ denote the internal side of the boundary surface and the far-field, respectively, $s_{\text {bdy }}$ represents the speed of sound at the boundary, $\mathbf{V}_{\infty}=\left(u_{\infty}, v_{\infty}, w_{\infty}\right)$ is the far-field flow velocity vector, and $\mathbf{V}_{\text {ref }}=\left(u_{\text {ref }}, v_{\text {ref }}, w_{\text {ref }}\right)$ is the reference velocity vector, as defined below.

(a) For $\mathbf{V}_{\infty} \cdot \mathbf{n}_{\text {bdy }}<0$, the inflow BC is defined, and then the parameters in Eqs.(39) and (40) are defined as

$$
\left\{\begin{array} { l } 
{ \rho _ { \mathrm { L } } = \rho _ { \mathrm { c } } + \nabla \rho _ { \mathrm { c } } \cdot \Delta \mathbf { r } _ { \mathrm { c } , \text { bdy } } } \\
{ u _ { \mathrm { L } } = u _ { \mathrm { c } } + \nabla u _ { \mathrm { c } } \cdot \Delta \mathbf { r } _ { \mathrm { c } , \text { bdy } } } \\
{ v _ { \mathrm { L } } = v _ { \mathrm { c } } + \nabla v _ { \mathrm { c } } \cdot \Delta \mathbf { r } _ { \mathrm { c } , \mathrm { bdy } } } \\
{ w _ { \mathrm { L } } = w _ { \mathrm { c } } + \nabla w _ { \mathrm { c } } \cdot \Delta \mathbf { r } _ { \mathrm { c } , \mathrm { bdy } } } \\
{ p _ { \mathrm { L } } = p _ { \mathrm { c } } + \nabla p _ { \mathrm { c } } \cdot \Delta \mathbf { r } _ { \mathrm { c } , \text { bdy } } }
\end{array} \quad \text { and } \quad \left\{\begin{array}{c}
\rho_{\text {ref }}=\rho_{\infty} \\
u_{\mathrm{ref}}=u_{\infty} \\
v_{\text {ref }}=v_{\infty} \\
w_{\text {ref }}=w_{\infty}
\end{array}\right.\right.
$$

where subscripts $\mathrm{c}$ denotes the center of the neighboring cell of the boundary.

(b) For $\mathbf{V}_{\infty} \cdot \mathbf{n}_{\text {bdy }} \geq 0$, the outflow BC is defined, and then the parameters in Eqs.(39) and (40) are defined as

$$
\left\{\begin{array} { l } 
{ \rho _ { \mathrm { L } } = \rho _ { \mathrm { c } } + \nabla \rho _ { \mathrm { c } } \cdot \Delta \mathbf { r } _ { \mathrm { c } , \mathrm { bdy } } } \\
{ u _ { \mathrm { L } } = u _ { \mathrm { c } } + \nabla u _ { \mathrm { c } } \cdot \Delta \mathbf { r } _ { \mathrm { c } , \text { bdy } } } \\
{ v _ { \mathrm { L } } = v _ { \mathrm { c } } + \nabla v _ { \mathrm { c } } \cdot \Delta \mathbf { r } _ { \mathrm { c } , \mathrm { bdy } } } \\
{ w _ { \mathrm { L } } = w _ { \mathrm { c } } + \nabla w _ { \mathrm { c } } \cdot \Delta \mathbf { r } _ { \mathrm { c } , \mathrm { bdy } } } \\
{ p _ { \mathrm { L } } = p _ { \mathrm { c } } + \nabla p _ { \mathrm { c } } \cdot \Delta \mathbf { r } _ { \mathrm { c } , \mathrm { bdy } } }
\end{array} \quad \text { and } \quad \left\{\begin{array}{c}
\rho_{\text {ref }}=\rho_{\mathrm{L}} \\
u_{\mathrm{ref}}=u_{\mathrm{L}} \\
v_{\mathrm{ref}}=v_{\mathrm{L}} \\
w_{\text {ref }}=w_{\mathrm{L}}
\end{array} .\right.\right.
$$

\section{(2) Supersonic inflow BC}

For the supersonic inflow BC, the variables at the boundary are only determined by the parameters at the far-field, and thus we have

$$
\left\{\begin{array}{l}
\rho_{\text {bdy }}=\rho_{\infty} \\
u_{\text {bdy }}=u_{\infty} \\
v_{\text {bdy }}=v_{\infty} \\
w_{\text {bdy }}=w_{\infty} \\
p_{\text {bdy }}=p_{\infty}
\end{array}\right.
$$




\section{(3) Supersonic outflow BC}

For the supersonic outflow BC, the variables at the boundary are only determined by the parameters at the boundary cell, and thus we have

\section{(4) Slip wall BC}

$$
\left\{\begin{array}{l}
\rho_{\text {bdy }}=\rho_{\mathrm{c}}+\nabla \rho_{\mathrm{c}} \cdot \Delta \mathbf{r}_{\mathrm{c}, \mathrm{bdy}} \\
u_{\text {bdy }}=u_{\mathrm{c}}+\nabla u_{\mathrm{c}} \cdot \Delta \mathbf{r}_{\mathrm{c}, \text { bdy }} \\
v_{\text {bdy }}=v_{\mathrm{c}}+\nabla v_{\mathrm{c}} \cdot \Delta \mathbf{r}_{\mathrm{c}, \text { bdy }} \\
w_{\text {bdy }}=w_{\mathrm{c}}+\nabla w_{\mathrm{c}} \cdot \Delta \mathbf{r}_{\mathrm{c}, \mathrm{bdy}} \\
p_{\text {bdy }}=p_{\mathrm{c}}+\nabla p_{\mathrm{c}} \cdot \Delta \mathbf{r}_{\mathrm{c}, \mathrm{bdy}}
\end{array} .\right.
$$

For the Euler equations, the slip wall $\mathrm{BC}$ requires that the flow satisfies the non-penetration constraint, thus the variables at the boundary are determined by

$$
\left\{\begin{array} { l } 
{ \rho _ { \text { bdy } } = \rho _ { \mathrm { L } } } \\
{ u _ { \text { bdy } } = u _ { \mathrm { L } } - ( u _ { \mathrm { L } } n _ { x } + v _ { \mathrm { L } } n _ { y } + w _ { \mathrm { L } } n _ { z } ) n _ { x } } \\
{ v _ { \text { bdy } } = v _ { \mathrm { L } } - ( u _ { \mathrm { L } } n _ { x } + v _ { \mathrm { L } } n _ { y } + w _ { \mathrm { L } } n _ { z } ) n _ { y } } \\
{ w _ { \text { bdy } } = w _ { \mathrm { L } } - ( u _ { \mathrm { L } } n _ { x } + v _ { \mathrm { L } } n _ { y } + w _ { \mathrm { L } } n _ { z } ) n _ { z } } \\
{ p _ { \text { bdy } } = p _ { \mathrm { L } } }
\end{array} \quad \text { and } \quad \left\{\begin{array}{l}
\rho_{\mathrm{L}}=\rho_{\mathrm{c}}+\nabla \rho_{\mathrm{c}} \cdot \Delta \mathbf{r}_{\mathrm{c}, \mathrm{bdy}} \\
u_{\mathrm{L}}=u_{\mathrm{c}}+\nabla u_{\mathrm{c}} \cdot \Delta \mathbf{r}_{\mathrm{c}, \mathrm{bdy}} \\
v_{\mathrm{L}}=v_{\mathrm{c}}+\nabla v_{\mathrm{c}} \cdot \Delta \mathbf{r}_{\mathrm{c}, \mathrm{bdy}} \\
w_{\mathrm{L}}=w_{\mathrm{c}}+\nabla w_{\mathrm{c}} \cdot \Delta \mathbf{r}_{\mathrm{c}, \mathrm{bdy}} \\
p_{\mathrm{L}}=p_{\mathrm{c}}+\nabla p_{\mathrm{c}} \cdot \Delta \mathbf{r}_{\mathrm{c}, \mathrm{bdy}}
\end{array} .\right.\right.
$$

The difference between the present boundary solutions and the traditional method $[35,36]$ is that the present boundary solutions are linearly extrapolated from the centroid of boundary cells using the gradients iteratively reconstructed with boundary constraints, ensuring linear exactness and satisfying BCs. Here, only the BCs used in this paper are introduced. Whereas, the other BCs, e.g. subsonic inflow/outflow BCs that impose the pressure, can also be defined straightforwardly.

\section{Numerical results of test functions}

In this section, we will discuss the performance of gradient reconstruction methods approximating one linear function and two nonlinear functions. Here, the relative error of the gradient is defined as

$$
\begin{aligned}
& e r r_{\text {rel }}=\frac{\nabla_{r} \varphi-\nabla_{\text {exact }} \varphi}{\nabla_{\text {exact }} \varphi} \\
& e r r_{\text {abs }}=\left|e r r_{\text {rel }}\right|
\end{aligned},
$$

where $\nabla_{r} \varphi$ and $\nabla_{\text {exact }} \varphi$ are the reconstructed gradient and the exact gradient, respectively. Here we use four types of grids to examine the convergence rate during mesh refinement. The grid types are: (I) quadrilateral (rectangular) grids; (II) regular triangular grids which are produced by diagonally splitting the cells in the quadrilateral grids; (III) irregular triangular grids which are produced by diagonally splitting the cells in the quadrilateral grids in random directions; and (IV) perturbed triangle grids which are produced by applying random perturbations to the vertexes of irregular triangle grids. The coarsest grid of each grid type is shown in Fig.6.

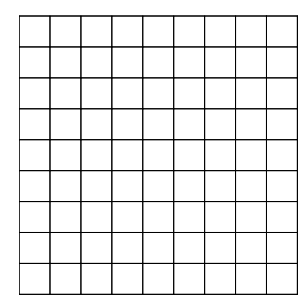

(a) Quadrilateral

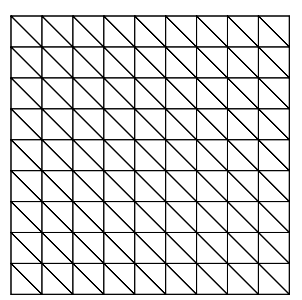

(b) Regular triangular

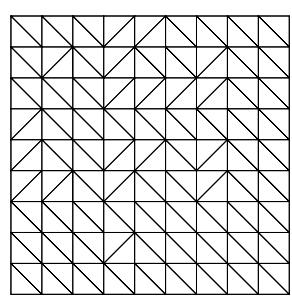

(c) Irregular triangular

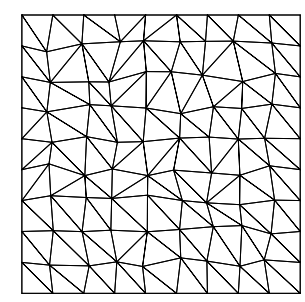

(d) Perturbed triangular

Fig. 6. Four grid types for testing grid convergence.

The $L_{\infty}$-norm and $L_{2}$-norm of the gradient error are calculated by

$$
\begin{aligned}
& \varepsilon_{L_{\infty}(\Omega)}=\left(\left\|\frac{\nabla_{r} \varphi-\nabla_{\text {exact }} \varphi}{\nabla_{\text {exact }}}\right\|_{\infty}\right)_{\Omega} \\
& \varepsilon_{L_{2}(\Omega)}=\frac{\left(\left\|\nabla_{r} \varphi-\nabla_{\text {exact }} \varphi\right\|_{2}\right)_{\Omega}}{\left(\left\|\nabla_{\text {exact }} \varphi\right\|_{2}\right)_{\Omega}}
\end{aligned}
$$


and the order of accuracy is calculated by

$$
p^{\text {ord }}=\frac{\log \frac{\varepsilon^{k^{1}}}{\varepsilon^{k^{1}+1}}}{\log \frac{h_{x}^{k^{k}}}{h_{x}^{k^{k}+1}}},
$$

where $h_{x}$ is the scale of the grid in the $x$ - or $y$-direction, and $k^{1}$ denotes the refinement level of the grids.

\subsection{Linear function}

In this section, a linear test function

$$
\varphi=1+3 x+2000 y
$$

is used to discuss the accuracy of the above methods for linear functions. In this case, the computational domain is a rectangular box of $[0.05,1.05] \times[0,0.001]$ (thus the aspect-ratio is 1000 ), the $19 \times 19$ (I) type grid and the (IV) type grid are used. The numerical errors of the reconstructed gradients on two grids are shown in Table 2 and Table 3 , respectively. On the quadrilateral grid, all the methods are exact for the linear function in the internal cells whose vertexes are not boundary vertexes. However, only the WLSQ-IC and VWLSQ-ITER methods are exact for the boundary cells adjacent to at least one boundary vertex, and the maximum errors of the GG methods and the WLSQGC method reach 50\%, because the boundary face solutions or the ghost cell solutions are not linearly extrapolated, losing the linear exactness. On the perturbed triangular grid, due to the same reason, GG methods and the WLSQ-GC method produce significant error. Since the internal grid cells are not regular or symmetry, the GG methods lose linear exactness even in the inner region. The present VWLSQ-ITER method is exact for the linear function on these two grids, which means that the iterative procedure is able to calculate both the gradients in boundary cells and the solutions at boundary faces accurately.

\begin{tabular}{|c|c|c|c|c|}
\hline \multirow{2}{*}{ Method } & \multicolumn{2}{|c|}{ Internal cells } & \multicolumn{2}{|c|}{ Boundary cells } \\
\hline & $\varepsilon_{L_{\infty}}\left(\frac{\partial \varphi}{\partial x}\right)$ & $\varepsilon_{L_{\infty}}\left(\frac{\partial \varphi}{\partial y}\right)$ & $\varepsilon_{L_{\infty}}\left(\frac{\partial \varphi}{\partial x}\right)$ & $\varepsilon_{L_{\infty}}\left(\frac{\partial \varphi}{\partial y}\right)$ \\
\hline CAGG & $9.50010736744 \mathrm{E}-10$ & $9.50013145484 \mathrm{E}-10$ & $4.99999999525 \mathrm{E}-01$ & $5.00000000000 \mathrm{E}-01$ \\
\hline NAGG-IDW & $1.21384384026 \mathrm{E}-14$ & $9.50017010837 \mathrm{E}-10$ & $5.00000000158 \mathrm{E}-01$ & $5.00000000000 \mathrm{E}-01$ \\
\hline NAGG-PLW & $1.00660220899 \mathrm{E}-14$ & $1.72803993337 \mathrm{E}-14$ & $5.00000000000 \mathrm{E}-01$ & $5.00000000000 \mathrm{E}-01$ \\
\hline WLSQ(0)-GC & $2.96059473233 \mathrm{E}-15$ & $3.86535248253 \mathrm{E}-15$ & 4.99999999525E-01 & $5.00000000000 \mathrm{E}-01$ \\
\hline WLSQ $(0)-I C$ & $2.96059473233 \mathrm{E}-15$ & $3.86535248253 \mathrm{E}-15$ & $3.70074341542 \mathrm{E}-15$ & $5.22959453519 \mathrm{E}-15$ \\
\hline VWLSQ(0-0)-ITER & $2.96059473233 \mathrm{E}-15$ & $6.82121026330 \mathrm{E}-15$ & $1.92438657602 \mathrm{E}-14$ & $3.70619090972 \mathrm{E}-14$ \\
\hline
\end{tabular}

Table 2. Numerical errors on the quadrilateral grid.

Table 3. Numerical errors on the perturbed triangle grid.

\begin{tabular}{cccccc}
\hline \multirow{2}{*}{ Method } & \multicolumn{2}{c}{ Internal cells } & & \multicolumn{2}{c}{ Boundary cells } \\
\cline { 2 - 3 } \cline { 5 - 6 } & $\varepsilon_{L_{\infty}}\left(\frac{\partial \varphi}{\partial x}\right)$ & $\varepsilon_{L_{\infty}}\left(\frac{\partial \varphi}{\partial y}\right)$ & & $\varepsilon_{L_{\infty}}\left(\frac{\partial \varphi}{\partial x}\right)$ & $\varepsilon_{L_{\infty}}\left(\frac{\partial \varphi}{\partial y}\right)$ \\
\hline CAGG & $1.35859491810 \mathrm{E}+00$ & $1.70724031795 \mathrm{E}+00$ & & $1.05749132332 \mathrm{E}+00$ & $1.60050949048 \mathrm{E}+00$ \\
NAGG-IDW & $6.44128871607 \mathrm{E}-01$ & $1.03080636577 \mathrm{E}+00$ & & $6.75931470688 \mathrm{E}-01$ & $8.36333811765 \mathrm{E}-01$ \\
NAGG-PLW & $4.25217549818 \mathrm{E}-02$ & $5.24449823526 \mathrm{E}-02$ & & $5.556665555667 \mathrm{E}-01$ & $8.08748536680 \mathrm{E}-01$ \\
WLSQ(0)-GC & $8.14163551392 \mathrm{E}-15$ & $1.04591890704 \mathrm{E}-14$ & & $9.45150465612 \mathrm{E}-01$ & $1.086291444444 \mathrm{E}+00$ \\
WLSQ(0)-IC & $8.14163551392 \mathrm{E}-15$ & $1.04591890704 \mathrm{E}-14$ & & $5.62512999143 \mathrm{E}-15$ & $8.52651282912 \mathrm{E}-15$ \\
VWLSQ(0-0)-ITER & $7.25345709422 \mathrm{E}-15$ & $1.35287336889 \mathrm{E}-14$ & & $4.30766533555 \mathrm{E}-14$ & $6.95763446856 \mathrm{E}-14$ \\
\hline
\end{tabular}

\subsection{Non-linear function}

In this section, a nonlinear test function

$$
\varphi=x^{2} e^{y}
$$

is used to examine the basic behaviours of the present method, and several typical commonly used methods. In this case, the computational domain is a rectangular box of $[0.0985,0.1015] \times[0.0985,0.1015]$. Therefore in this case the aspect ratio of the quadrilateral cells is 1 .

We first discuss the gradient accuracy in internal cells whose vertexes are not boundary nodes, as shown in Figs.710. Therefore, the iterative strategy is not applied, and thus the results of VWLSQ methods are shown in the figures. Moreover, since on quadrilateral grids the inverse distance weights do not change the results, only the non-weighted 
methods are compared for quadrilateral grids. For internal cells, only the $L_{\infty}$ error is presented for simplicity, since the $L_{2}$ error convergence is similar.

It can be seen that all the (V)WLSQ methods and the NAGG-PLW method show second-order accurate results of the gradients on the quadrilateral grids. However, the NAGG-IDW method and the CAGG method show only the first-order accurate results on the same grids. It must be noted that the second-order accurate gradients are produced only because the stencils are symmetry/regular and so error cancellations occur. Obviously, the accuracy of NA-type methods depends on the accuracy of nodal solutions, i.e. IDW or PL weight. On triangular grids all the (V)WLSQ methods can achieve first-order gradient accuracy, but the computational error of the VWLSQ method is smaller than those of the WLSQ method. Especially on regular triangular grids, only the VWLSQ method shows second-order convergence, which is, again, due to the symmetry of the gradient stencils at a vertex. On irregular triangle grids and perturbed triangle grids, the GG-type methods have lost accuracy (except the NAGG-PLW method on irregular grids without perturbation). Moreover, the VWLSQ method without using the IDW in the averaging-gradient process, i.e. VWLSQ(0/1-0), is more accurate on regular triangular grids. In general one may conclude that the VWLSQ method consistently provide more accurate gradients in these test cases.

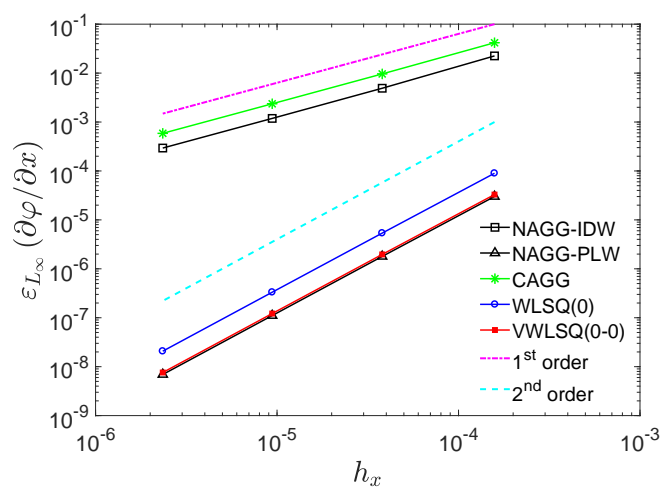

(a) $x$-direction

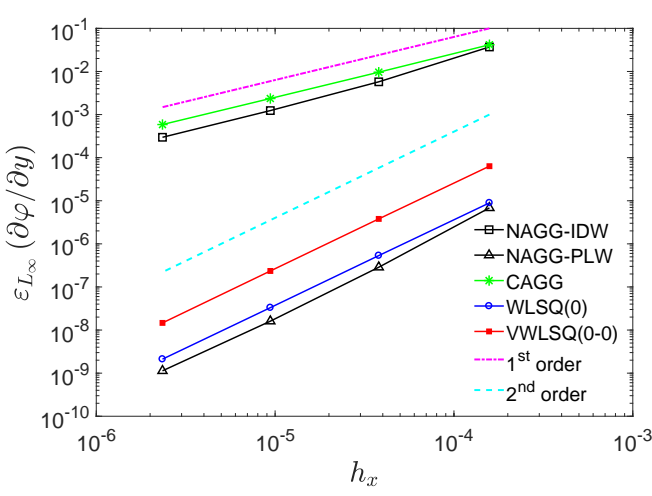

(b) $y$-direction

Fig. 7. The $L_{\infty}$-norm of the gradient error on quadrilateral grids with the test function $\varphi=x^{2} e^{y}$ : internal cells.

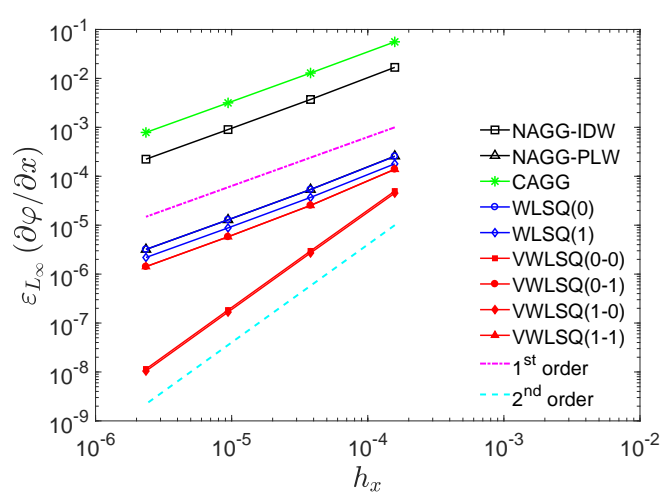

(a) $x$-direction

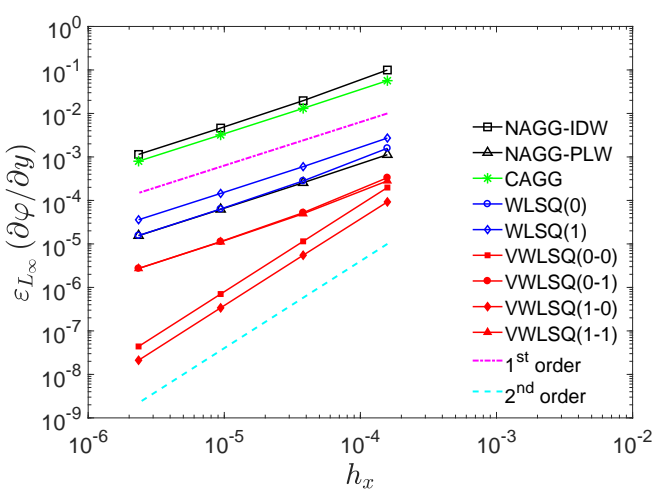

(b) $y$-direction

Fig. 8. The $L_{\infty}$-norm of the gradient error on regular triangular grids with the test function $\varphi=x^{2} e^{y}$ : internal cells.

The gradient error in boundary cells which connect with at least one boundary vertex is shown in Figs.11-14. Again, the $L_{2}$ errors are not shown for simplicity. The present iterative strategy is activated in the calculations. Except the present VWLSQ-ITER method and the WLSQ-IC method, none of the other methods can provide first-order accurate gradients for boundary cells. This is because the ghost cell solutions or boundary face solutions calculated by the first-order extrapolation are not exact for linear functions, and the spatial reconstructions depending on these stencils are not longer first-order accurate for the gradients. Moreover, the IDW in the present VWLSQ-ITER method 


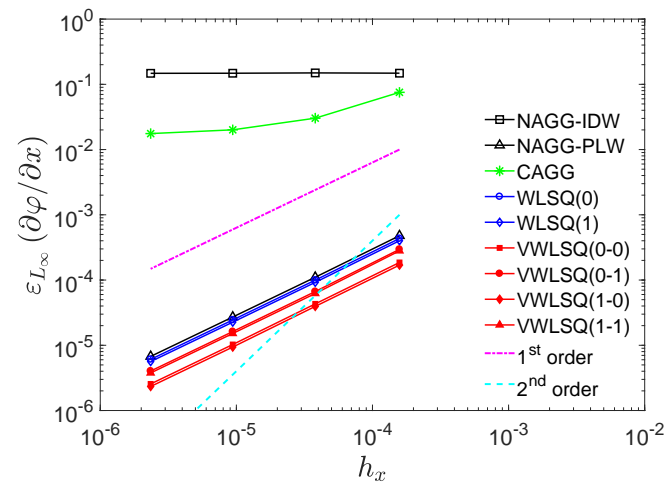

(a) $x$-direction

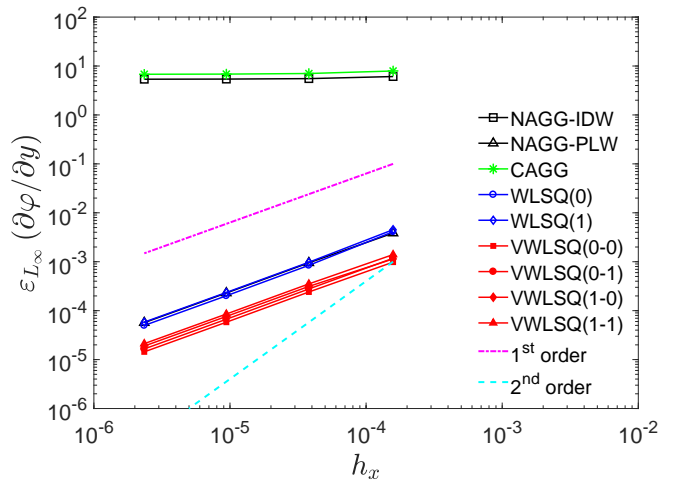

(b) $y$-direction

Fig. 9. The $L_{\infty}$-norm of the gradient error on irregular triangular grids with the test function $\varphi=x^{2} e^{y}$ : internal cells.

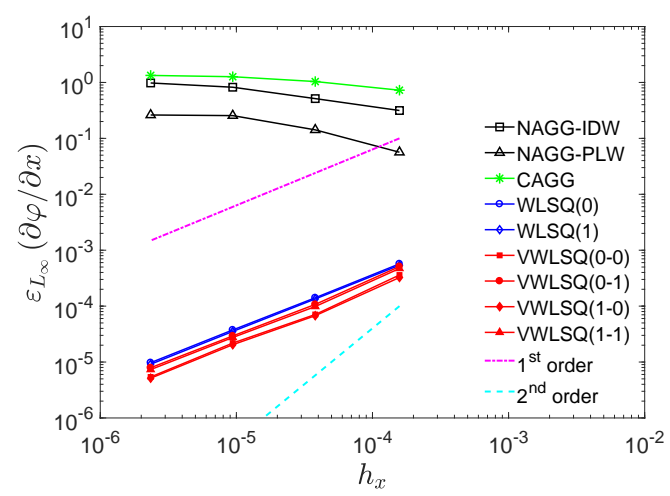

(a) $x$-direction

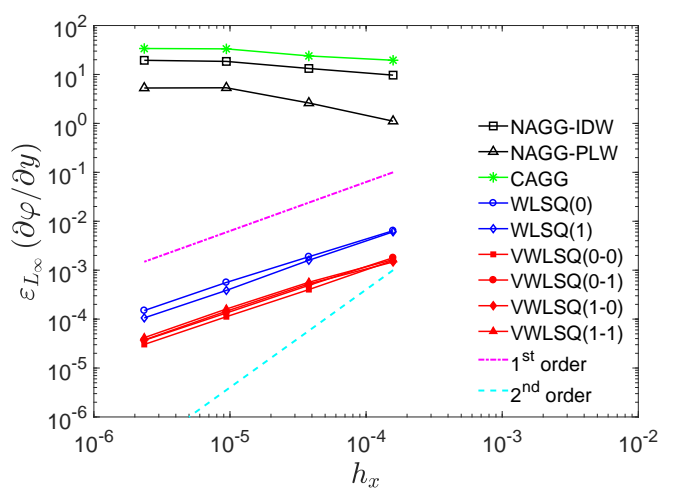

(b) $y$-direction

Fig. 10. The $L_{\infty}$-norm of the gradient error on perturbed triangular grids with the test function $\varphi=x^{2} e^{y}$ : internal cells. 
shows little influence on these near-boundary reconstruction results, probably because the iterative strategy has more significant influence in this scenario. In general, the present VWLSQ-ITER method ensures that the gradients in the boundary cells are first-order accurate, and also updates the boundary solutions accordingly with taking the boundary conditions into account.

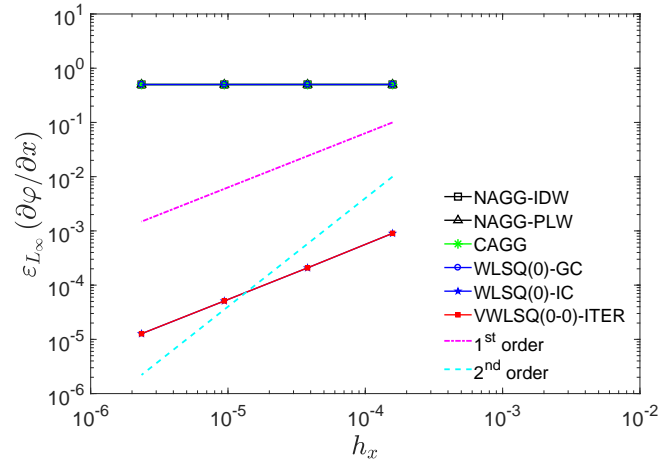

(a) $x$-direction

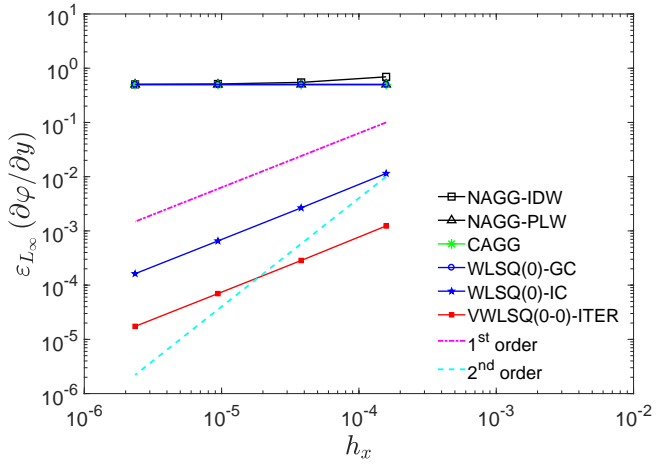

(b) $y$-direction

Fig. 11. The $L_{\infty}$-norm of the gradient error on quadrilateral grids with the test function $\varphi=x^{2} e^{y}$ : boundary cells.

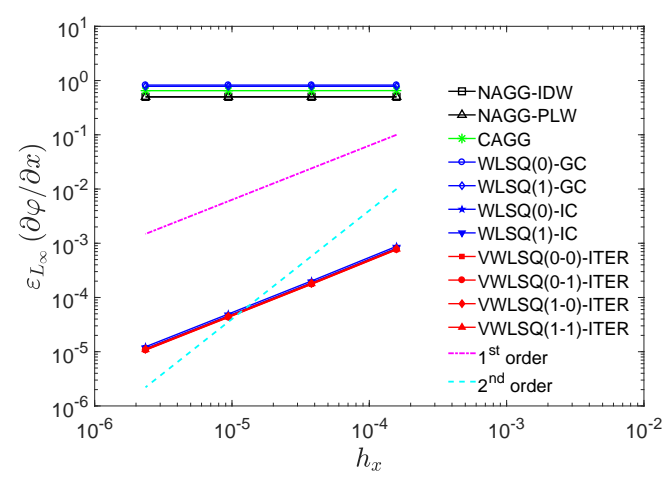

(a) $x$-direction

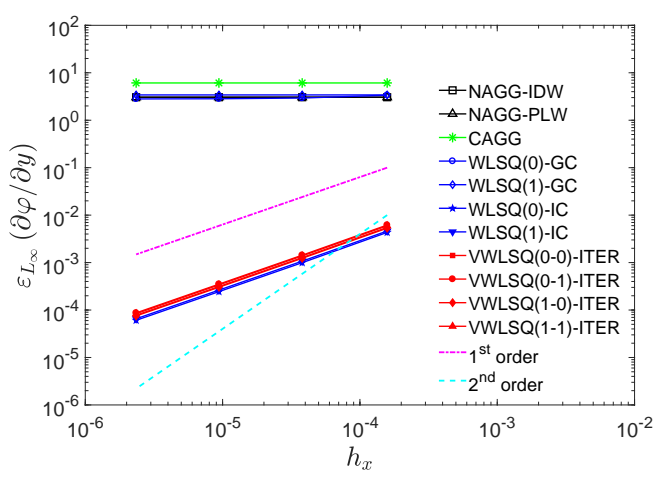

(b) $y$-direction

Fig. 12. The $L_{\infty}$-norm of the gradient error on regular triangular grids with the test function $\varphi=x^{2} e^{y}$ : boundary cells.

\subsection{Advection-diffusion equation: method of manufactured solution}

In order to test the present methods with more realistic problems, the method of manufactured solutions (MMS) [52] is applied, which is an effective way to evaluate the accuracy of spatial discretization methods [53, 54]. In this section, the manufactured solution of the advection-diffusion equation shown in Ref.[55] is used as a test case to verify the accuracy of the gradient reconstruction methods. The computational domain is a rectangular box of $[0.05,1.05] \times[0,0.001]$. The solution has the form of

$$
\varphi=1-e^{\frac{-y}{\sqrt{c \mu x}}},
$$

where $c=0.59$ and $\mu=10^{-8}$. The distribution of the solution mimics boundary layer flows, as shown in Fig.15. In this case, the four types of meshes shown in Fig.6 are used again. However, in this case the aspect ratio of each cell is 1000. It should be emphasized that in order to avoid being disturbed by gradients close to zero, only the gradients satisfying $\left|\left(\frac{\partial \varphi}{\partial x}\right)_{\text {exact }}\right|>10^{-5}$ are considered in the interior of this case.

The gradient accuracy in internal cells is shown in Figs.16-23, and the convergence is very different between different types of grids. On quadrilateral grids, eventually all the methods produce smaller error on finer meshes, 


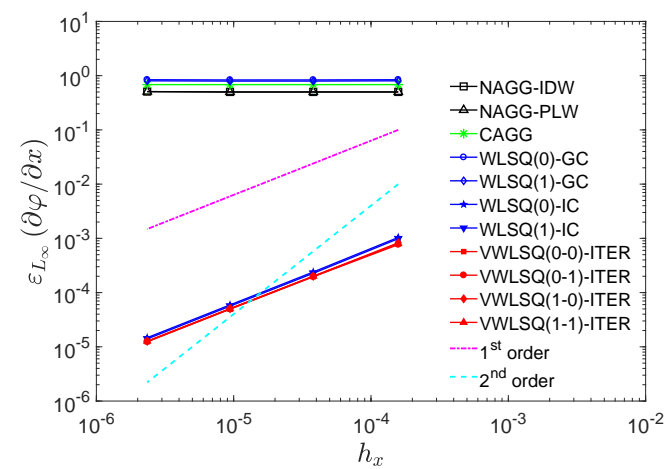

(a) $x$-direction

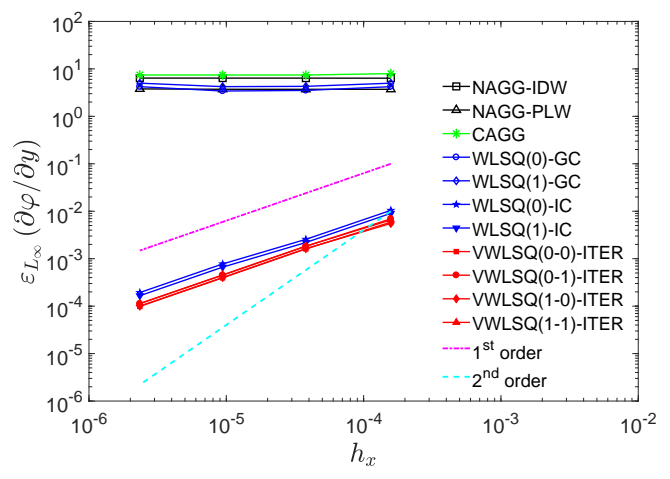

(b) $y$-direction

Fig. 13. The $L_{\infty}$-norm of the gradient error on irregular triangular grids with the test function $\varphi=x^{2} e^{y}$ : boundary cells.

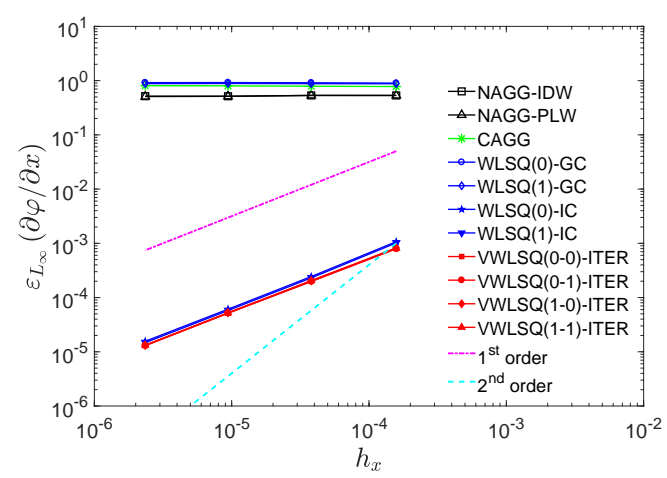

(a) $x$-direction

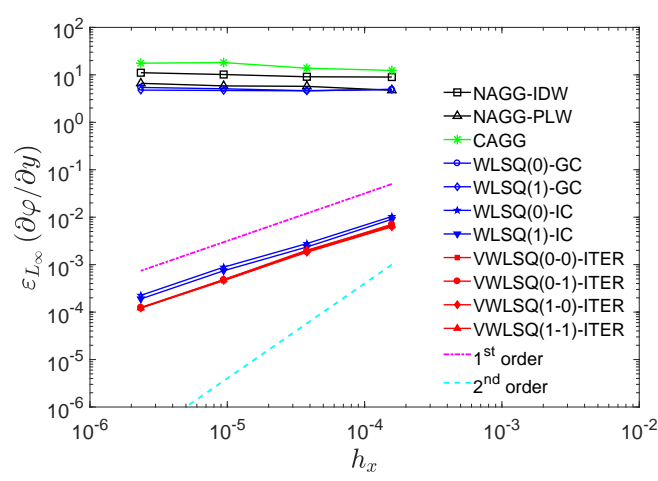

(b) $y$-direction

Fig. 14. The $L_{\infty}$-norm of the gradient error on perturbed triangular grids with the test function $\varphi=x^{2} e^{y}$ : boundary cells.

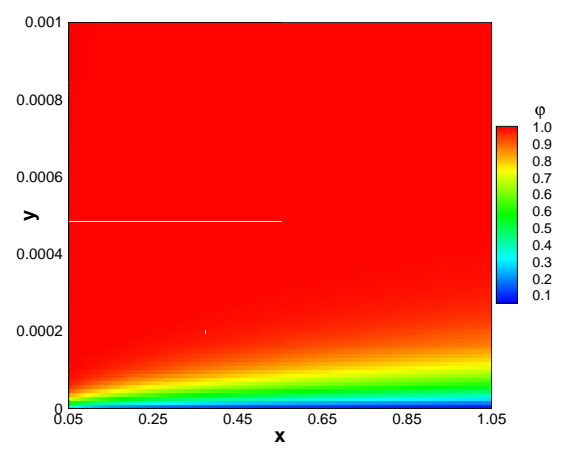

Fig. 15. Manufactured solution of the advection-diffusion equation. 
although the VWLSQ method is not the most accurate one. It should be noted that on quadrilateral meshes the VWLSQ method shows the same results as those of the other vertex-based methods including the NA-GG method using different weights. However, on triangular meshes the VWLSQ method shows the most accurate solutions compared with the other methods. Especially, except in the $x$-direction of the irregular triangular meshes, the VWLSQ method without using weighting in the averaging-gradient process, i.e. VWLSQ(0/1-0), always provides converged results. In general, although the VWLSQ method is not a silver bullet for computations on all types of meshes, it still usually shows more accurate solutions in challenging scenarios, e.g. computing on the irregular triangular meshes.

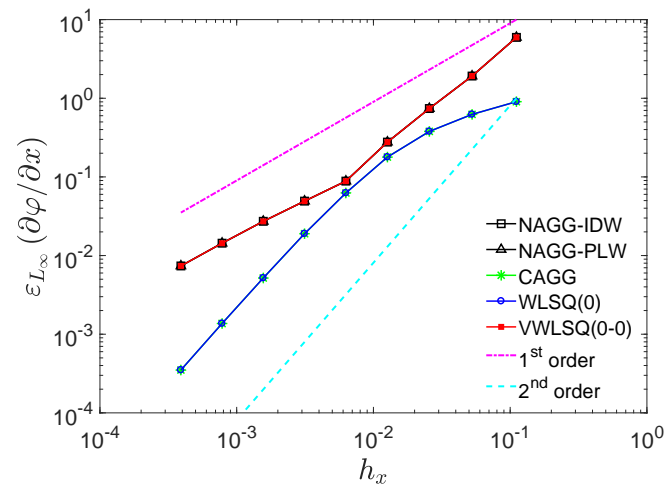

(a) $x$-direction

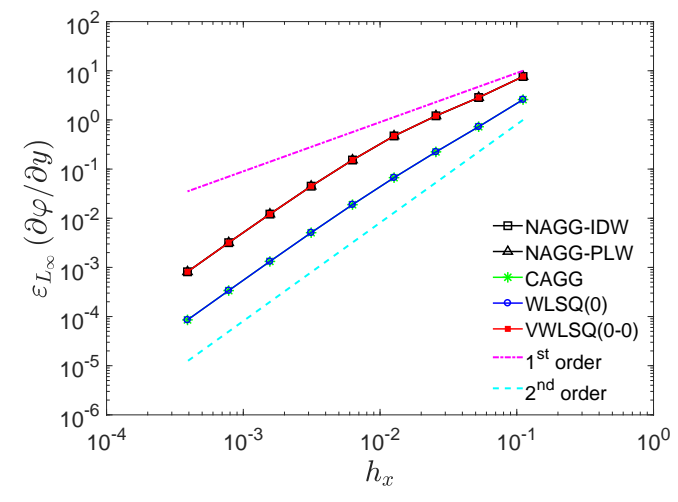

(b) $y$-direction

Fig. 16. The $L_{\infty}$-norm of the gradient error on quadrilateral grids discretizing the advection-diffusion equation: internal cells.

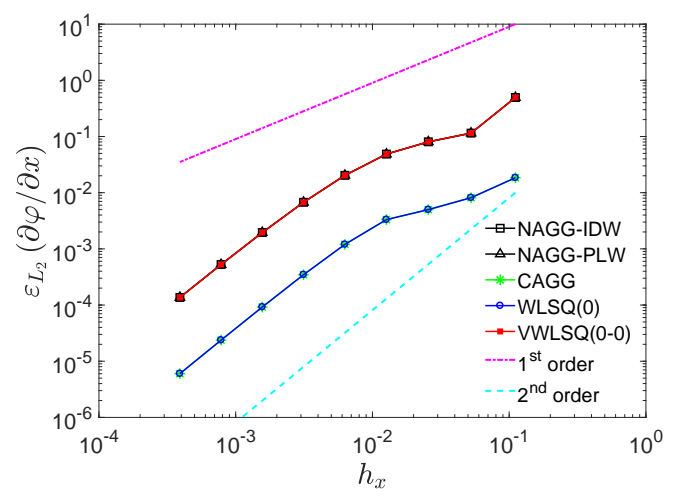

(a) $x$-direction

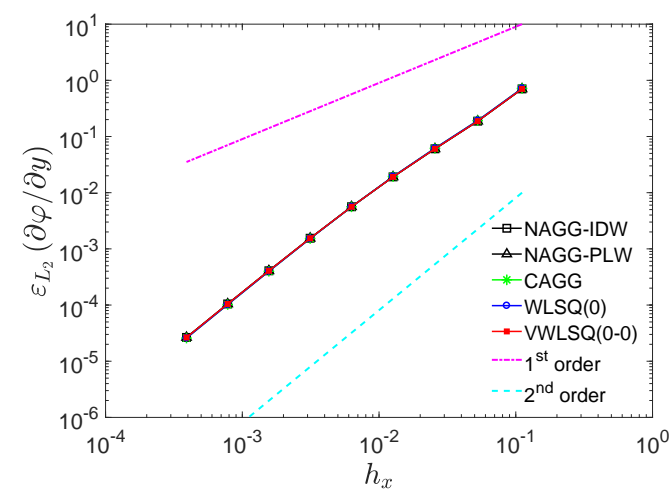

(b) $y$-direction

Fig. 17. The $L_{2}$-norm of the gradient error on quadrilateral grids discretizing the advection-diffusion equation: internal cells.

As in the previous case, the accuracy in boundary cells is also investigated in this case. However, it should be noted that in this case only the boundary cells near the lower boundary are investigated since the solutions of approximating the manufactured boundary layer need to be specifically investigated. Moreover, the results in the $x$-direction are also omitted since the significant variation in the $y$-direction is more suitable to show the difference. The results are shown in Figs.24-27. In general, only the WLSQ-IC method and the present VWLSQ-ITER method are able to provide, nearly, first-order accurate gradients on all types of grids. The stencil of the WLSQ-IC method excludes the first-order accurate solutions in ghost cells, and thus the linear exactness can be guaranteed. The present VWLSQ-ITER method iteratively modifies the boundary solutions, and thus the gradients and the solutions (at boundary faces) are both exact for linear functions. 


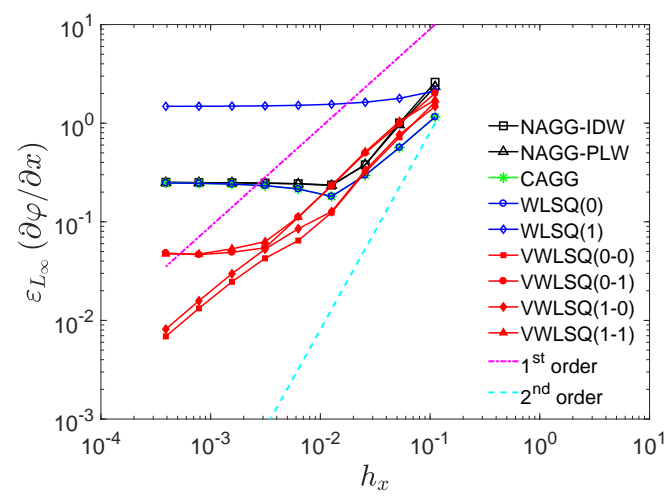

(a) $x$-direction

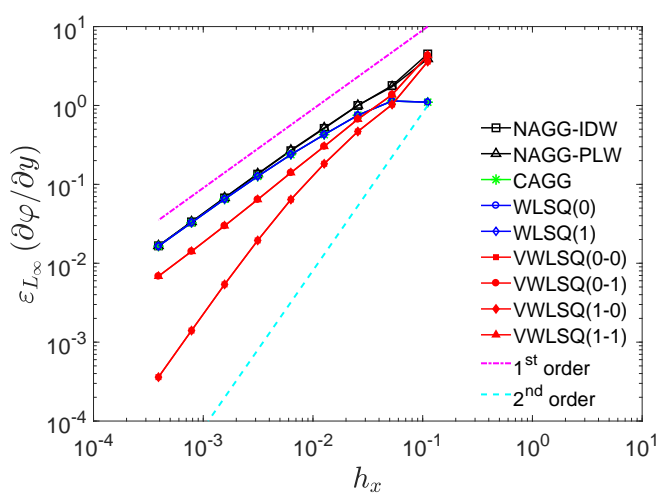

(b) $y$-direction

Fig. 18. The $L_{\infty}$-norm of the gradient error on regular triangular grids discretizing the advection-diffusion equation: internal cells.

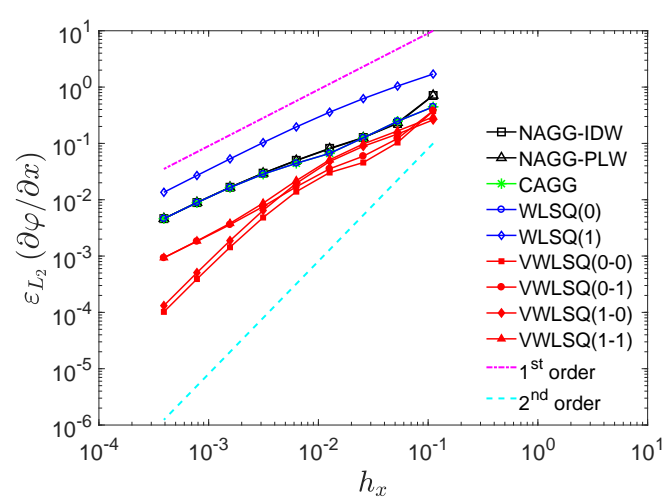

(a) $x$-direction

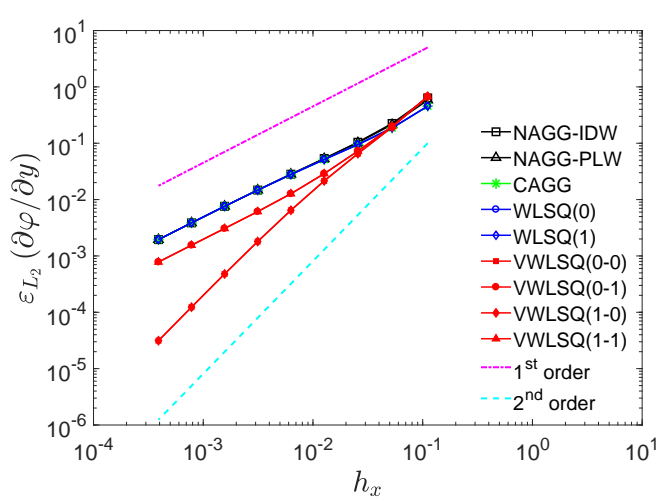

(b) $y$-direction

Fig. 19. The $L_{2}$-norm of the gradient error on regular triangular grids discretizing the advection-diffusion equation: internal cells.

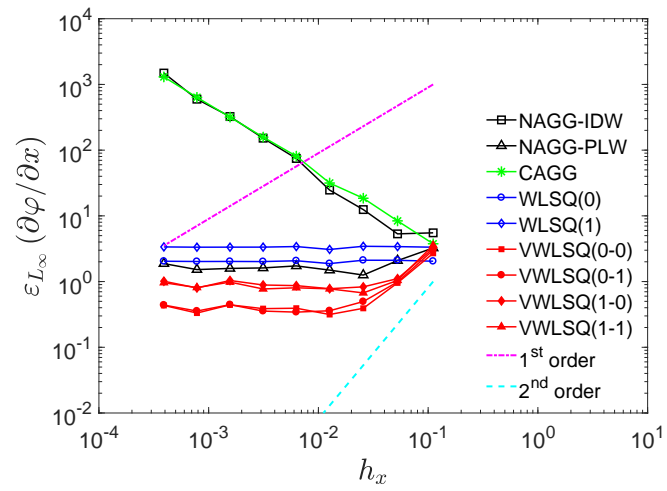

(a) $x$-direction

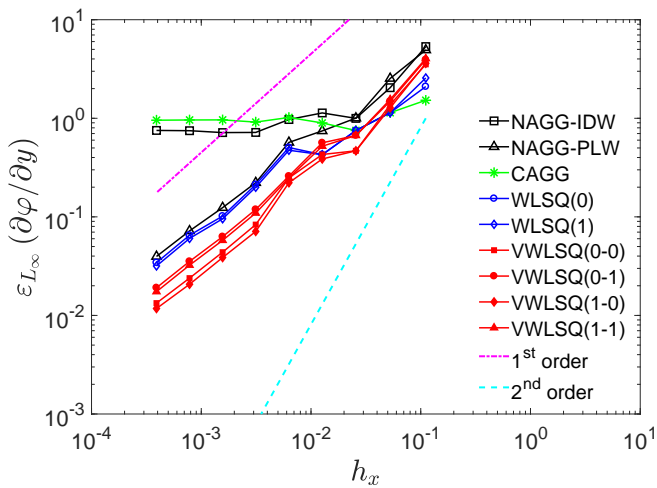

(b) $y$-direction

Fig. 20. The $L_{\infty}$-norm of the gradient error on irregular triangular grids discretizing the advection-diffusion equation: internal cells. 


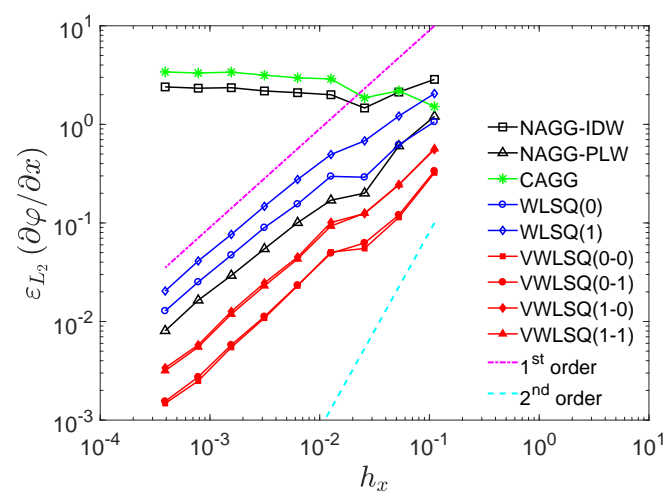

(a) $x$-direction

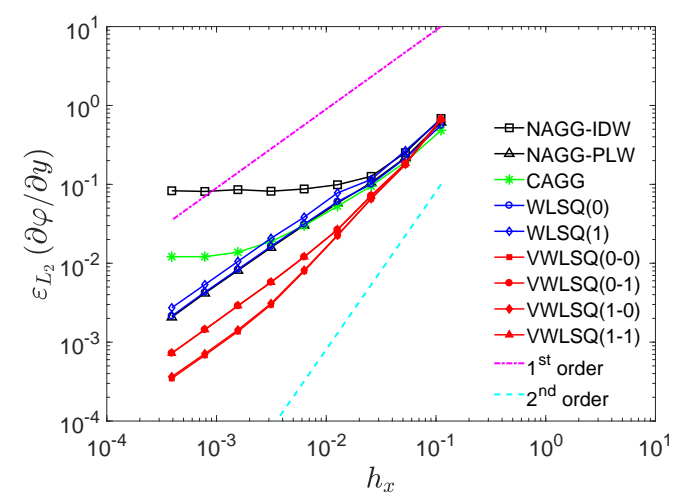

(b) $y$-direction

Fig. 21. The $L_{2}$-norm of the gradient error on irregular triangular grids discretizing the advection-diffusion equation: internal cells.

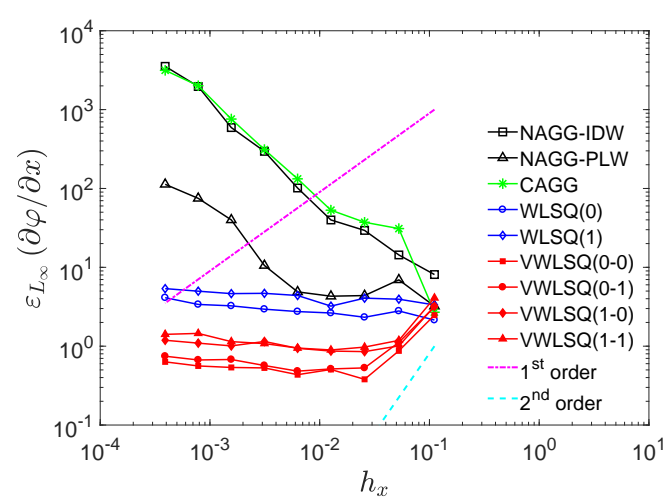

(a) $x$-direction

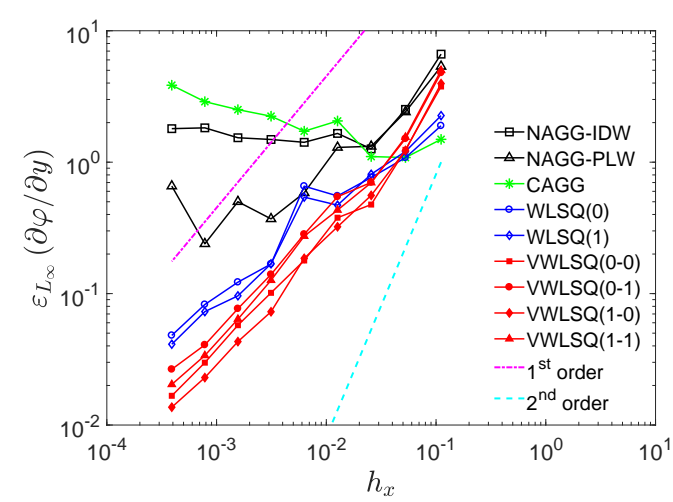

(b) $y$-direction

Fig. 22. The $L_{\infty}$-norm of the gradient error on perturbed triangular grids discretizing the advection-diffusion equation: internal cells.

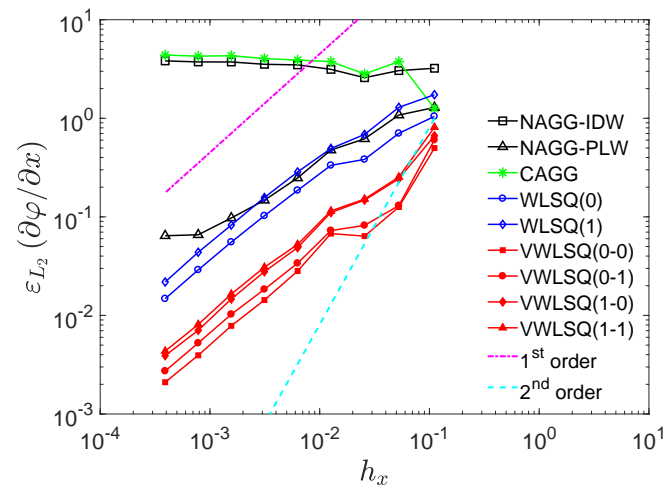

(a) $x$-direction

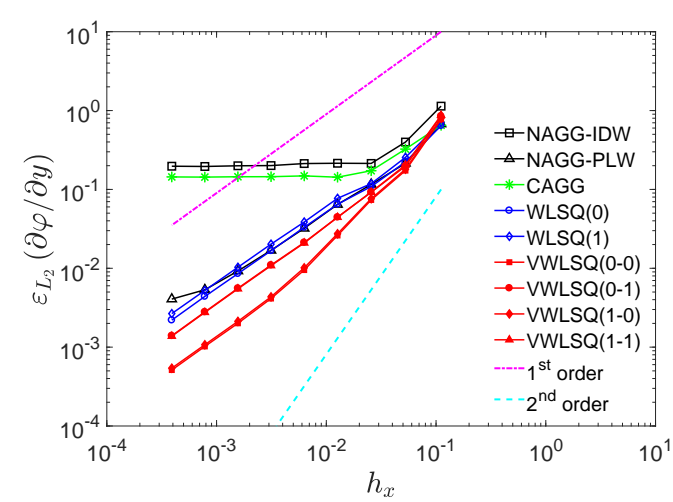

(b) $y$-direction

Fig. 23. The $L_{2}$-norm of the gradient error on perturbed triangular grids discretizing the advection-diffusion equation: internal cells. 


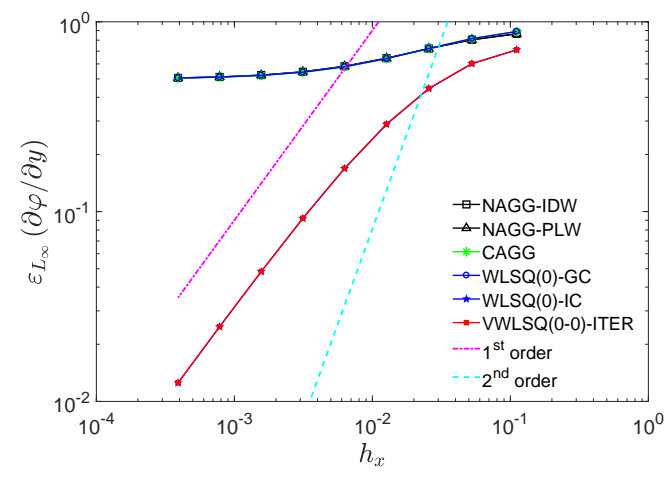

(a) $L_{\infty}$-norm

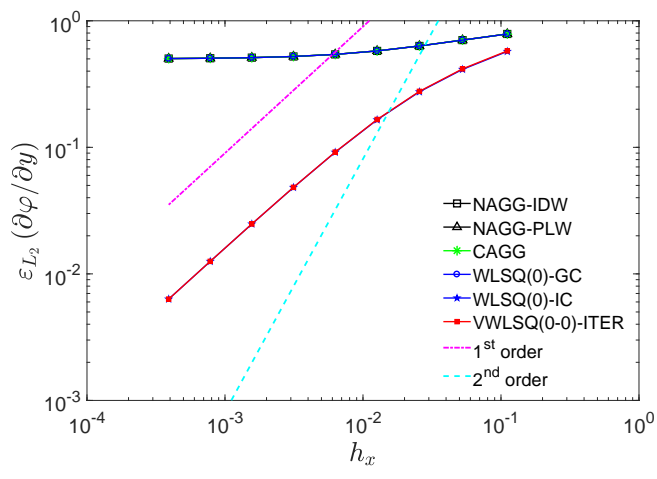

(b) $L_{2}$-norm

Fig. 24. The gradient error in the $y$-direction on quadrilateral grids discretizing the advection-diffusion equation: boundary cells.

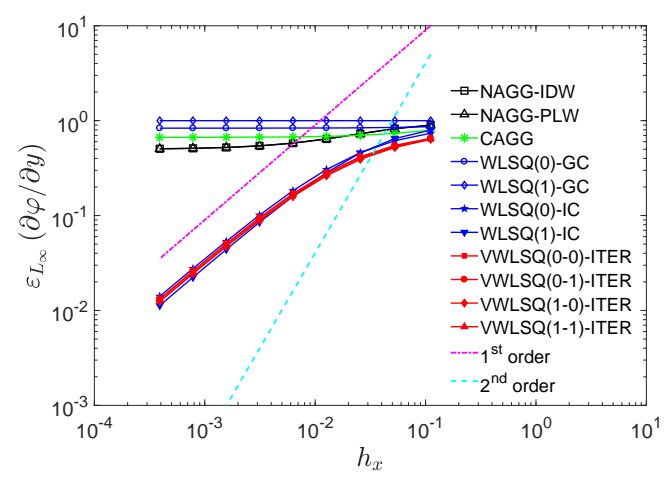

(a) $L_{\infty}$-norm

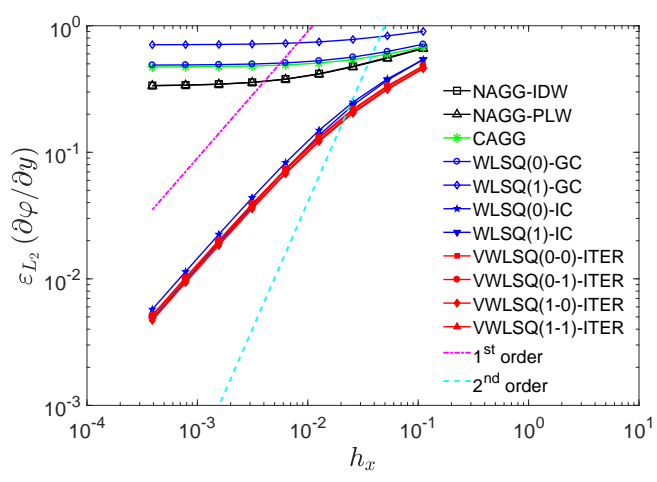

(b) $L_{2}$-norm

Fig. 25. The gradient error in the $y$-direction on regular triangular grids discretizing the advection-diffusion equation: boundary cells.

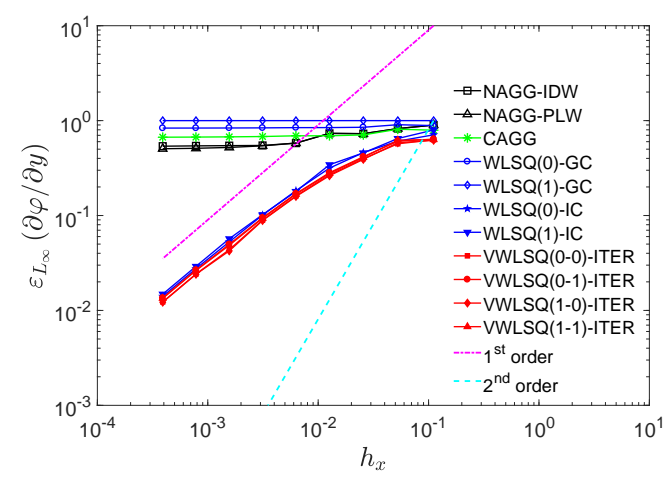

(a) $L_{\infty}$-norm

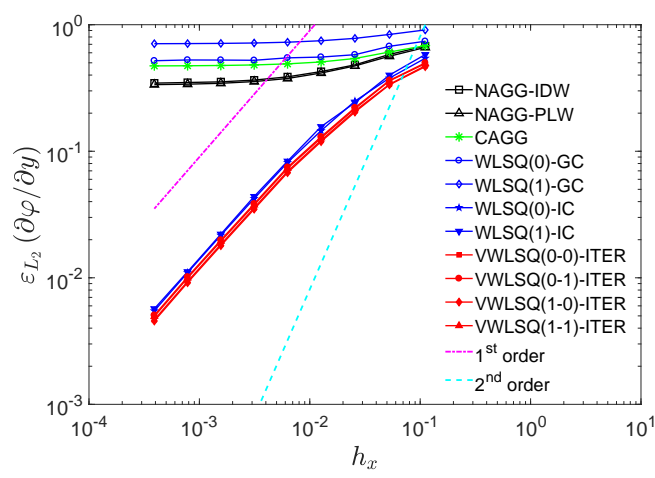

(b) $L_{2}$-norm

Fig. 26. The gradient error in the $y$-direction on irregular triangular grids discretizing the advection-diffusion equation: boundary cells. 


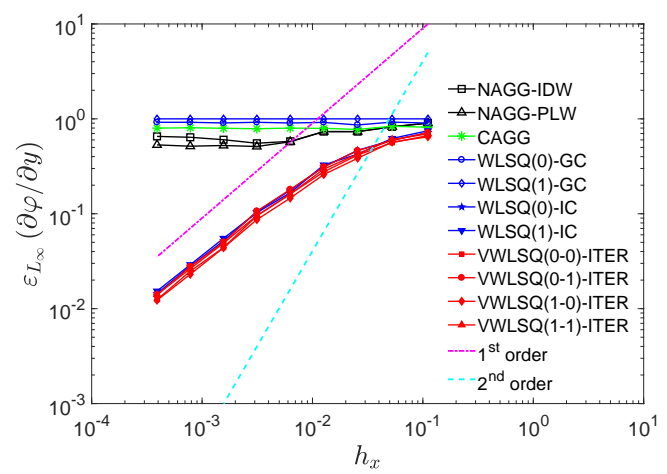

(a) $L_{\infty}$-norm

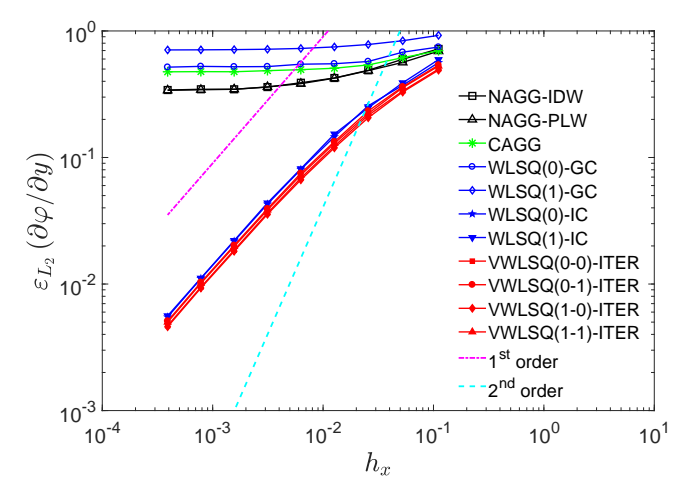

(b) $L_{2}$-norm

Fig. 27. The gradient error in the $y$-direction on perturbed triangular grids discretizing the advection-diffusion equation: boundary cells.

\section{Numerical results of flow simulations}

\subsection{Subsonic inviscid flows around a cylinder}

In this subsection, we use a subsonic inviscid flows case to examine the present method on flow fields with curving boundaries. The flow Mach number is 0.3 , and the angle of attack is 0 . Therefore, in this case theoretically there should be no drag for any obstacle. Here, a circular cylinder is set in the flow field, and thus for this simple geometry the discretizations are easily provided, as shown in Fig.28. The radius of the cylinder is 1, and a 399 (circumferential) $\times 50$ (radial) quadrilateral mesh and a triangular mesh of 52396 cells are used. Moreover, the HLLC scheme [39] is used as the numerical flux function, and the time step of the LU-SGS scheme is calculated based on CFL $=10$. The termination conditions $N_{\text {iter }}^{\max }$ and $\varepsilon$ of the present iterative strategy are respectively defined as 10 and $10^{-5}$.

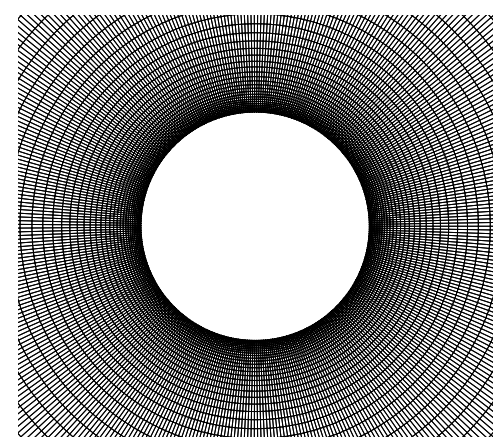

(a) Quadrilateral mesh

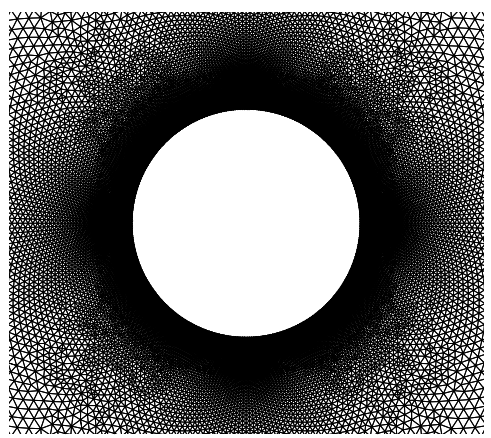

(b) Triangle mesh

Fig. 28. Grids around a circular cylinder.

The Mach number contours and entropy contours (defined as $\ln \left[\left(p / \rho^{\gamma}\right) /\left(p_{\infty} / \rho_{\infty}^{\gamma}\right)\right]$ ) on the quadrilateral mesh are shown in Fig.29 and Fig.30, respectively. In general, the Mach number contours of different methods are similar. However, the present method produces better results in the leeward side of the cylinder, leading to a symmetrical contour. Whereas, the other methods produce zig-zag shape contour lines in the leeward region. The reason causing this problem can be explained by the entropy contours. It is found that due to numerical dissipation, more entropy is produced around the cylinder by the traditional methods, eventually affecting the downstream numerical results. Whereas, the present VWLSQ-ITER method produces lower entropy, which means its overall numerical dissipation is lower than the other methods.

Moveover, the reason causing the difference between the present method and the WLSQ-IC method needs to be further addressed. As shown in previous results, the WLSQ-IC method is able to provide linearly-exact gradients in boundary cells. However, BCs are weakly imposed through numerical fluxes at boundary faces, which means that the gradient reconstruction procedure using the WLSQ-IC method doesn't directly take boundary constrains into 
account. In the meantime, in order to calculate numerical fluxes at boundary face quadrature points (face centroid), boundary solutions need to be given for the Weak-Prescribed approach [31], but the linear-exactness is not always provided while using a typical approach $[35,36]$ as mentioned previously. Whereas, as introduced in section 4.2 , the present method iteratively modify boundary cell gradients and boundary face solutions altogether to finally achieve linear-exactness for both internal and boundary numerical fluxes and to satisfy boundary constrains, and thus better accuracy may be attained by using the present method.

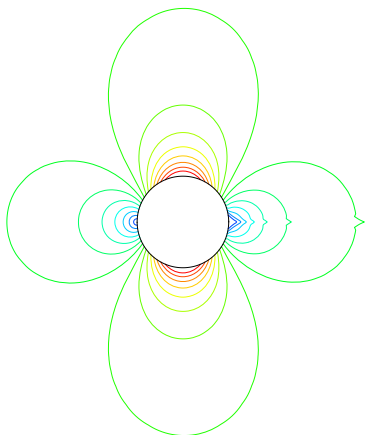

(a) CAGG

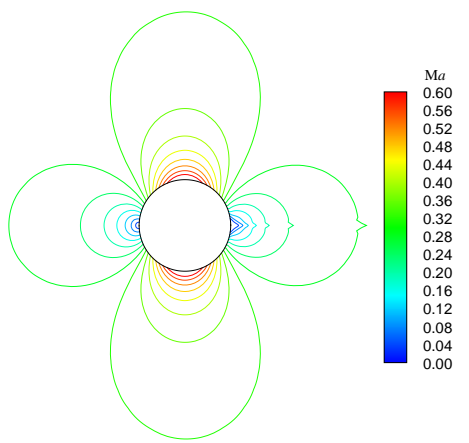

(d) WLSQ(1)-GC

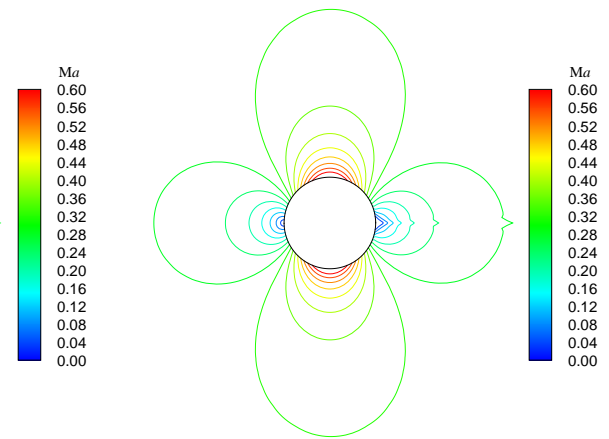

(b) NAGG-IDW

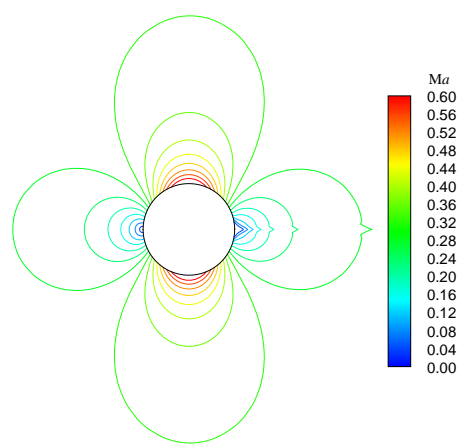

(e) WLSQ(1)-IC

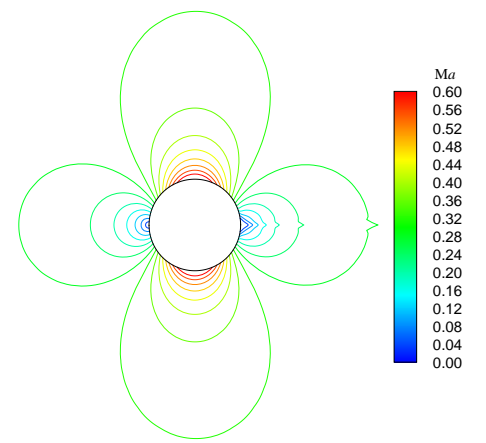

(c) NAGG-PLW

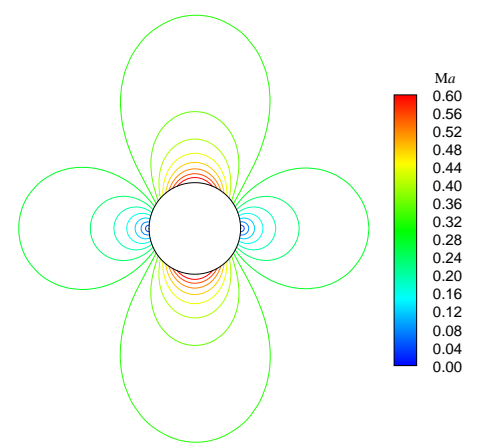

(f) VWLSQ(1-0)-ITER

Fig. 29. Mach number contours around the cylinder: quadrilateral mesh.

Similar behaviours can also be found on the triangular mesh. The Mach number contours and entropy contours on the triangle mesh are shown in Fig.31 and Fig.32, respectively. It can be found that the present VWLSQ-ITER method still performs well on the triangular mesh, the entropy is significantly reduced by the present method and the symmetrical Mach number contour is shown. Especially, the present method shows similar performance on both the quadrilateral and triangular meshes.

The drag coefficients calculated by using different methods are shown in Table 4. As mentioned above, theoretically the drag of any obstacle in incompressible inviscid flows shall be zero. However, the numerical methods we use have numerical error and thus zero drag cannot be guaranteed. In other word, an accurate numerical method should be able to provide numerical drag close to zero. Of course, the drag of the cylinder is an integral variable which cannot show the detail of the flow fields. In this case, the present VWLSQ-ITER method gives the smallest drag, which is an auxiliary evidence indicating that the VWLSQ-ITER method better recovers the linear exactness.

Table 4. Numerical drag coefficients $\left(C_{d}\right)$ of the cylinder.

\begin{tabular}{ccccccc}
\hline Grid type & CAGG & NAGG-IDW & NAGG-PLW & WLSQ(1)-GC & WLSQ(1)-IC & VWLSQ(1-0)-ITER \\
\hline Quadrilateral & 0.003187 & 0.002765 & 0.002672 & 0.003802 & 0.003885 & 0.002262 \\
Triangle & 0.003632 & 0.002570 & 0.002596 & 0.002607 & 0.002493 & 0.002263 \\
\hline
\end{tabular}

The number of iterations per time step is shown in Fig.33. It can be found that at the beginning of the computations the iteration procedure is invoked regularly. After about 3000 time steps, the iterative strategy is not activated since the near boundary region may be stable, although the computations are not fully converged. Therefore, we can conclude 


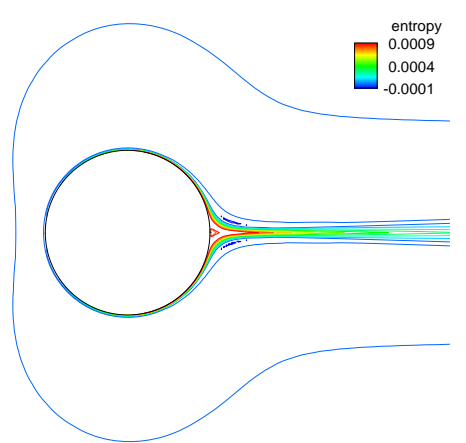

(a) CAGG

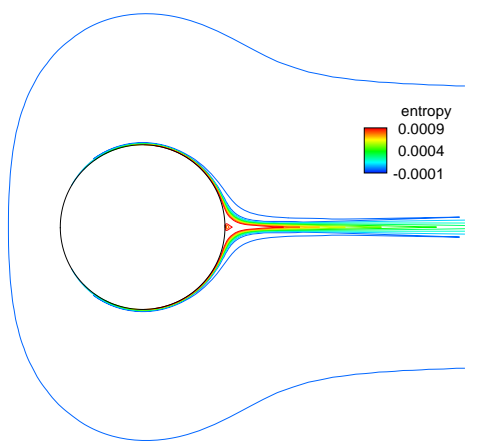

(d) WLSQ(1)-GC

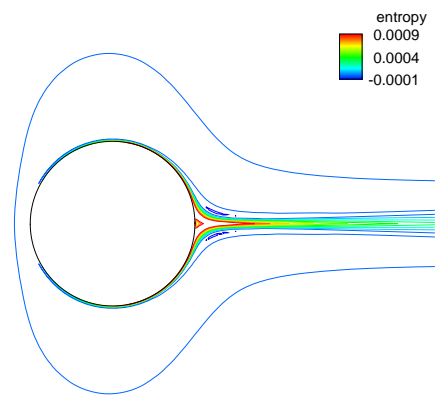

(b) NAGG-IDW

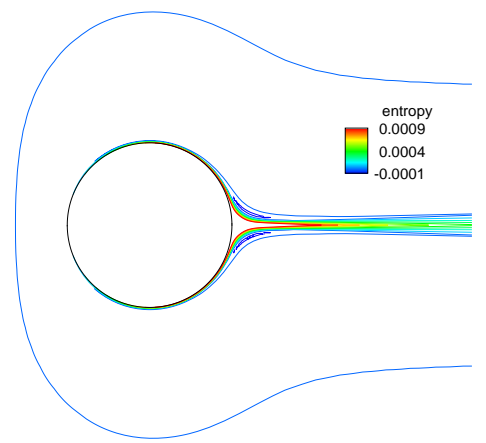

(e) WLSQ(1)-IC

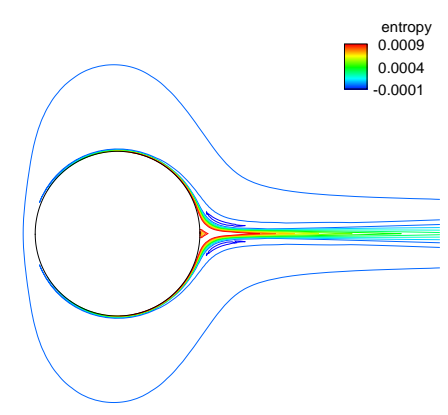

(c) NAGG-PLW

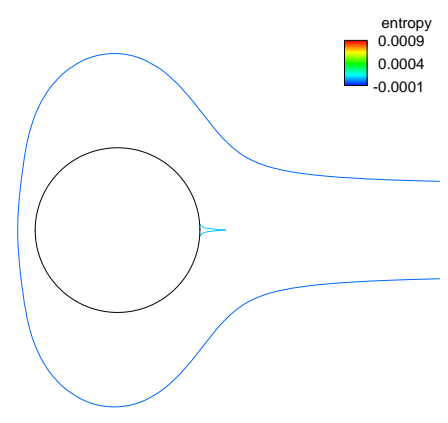

(f) VWLSQ(1-0)-ITER

Fig. 30. Entropy contours around the cylinder: quadrilateral mesh.

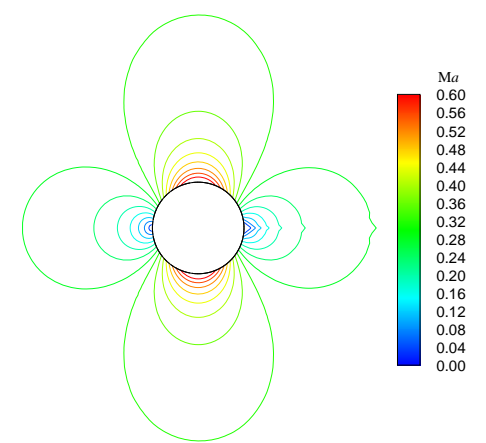

(a) CAGG

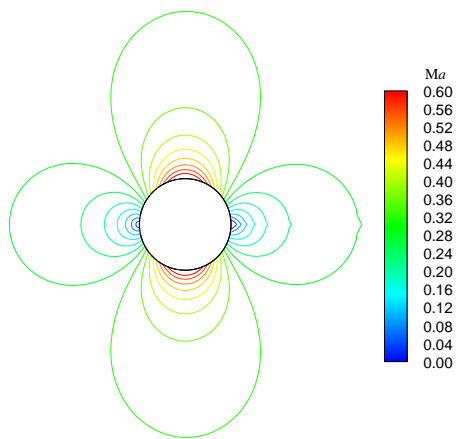

(d) WLSQ(1)-GC

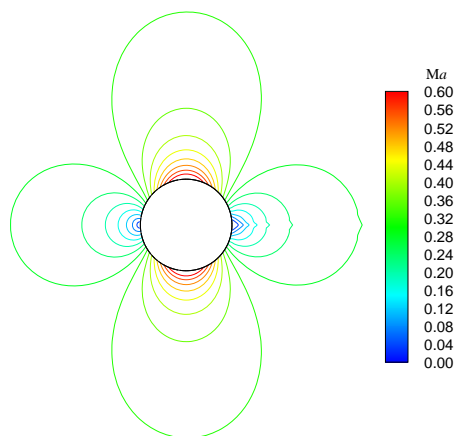

(b) NAGG-IDW

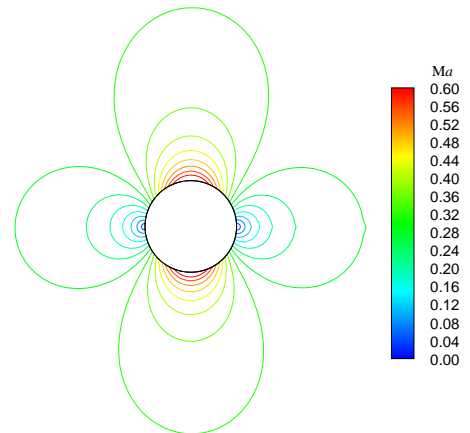

(e) WLSQ(1)-IC

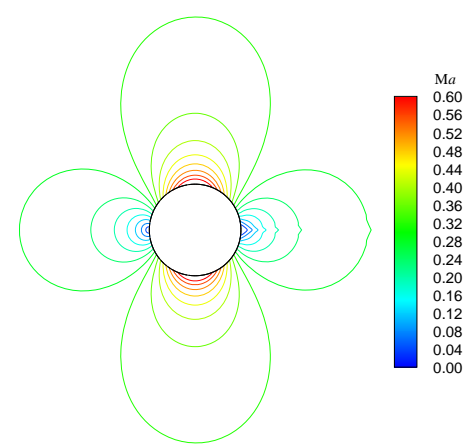

(c) NAGG-PLW

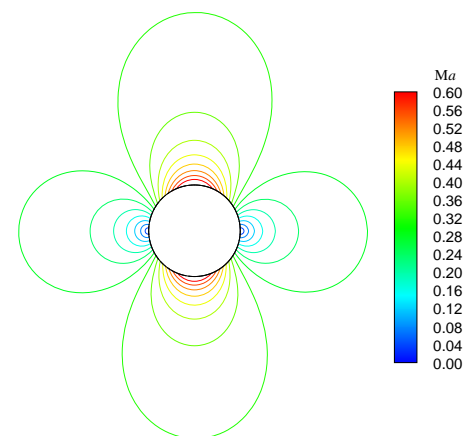

(f) VWLSQ(1-0)-ITER

Fig. 31. Mach number contours around the cylinder: triangle mesh. 


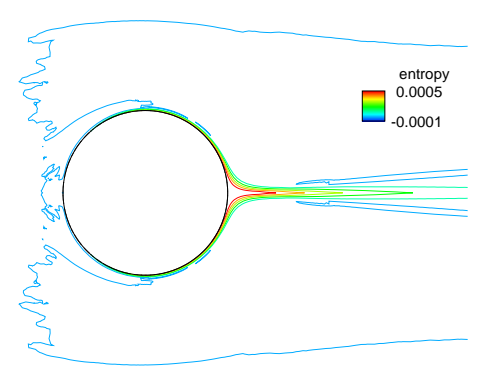

(a) CAGG

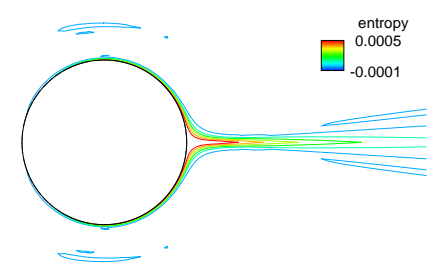

(d) WLSQ(1)-GC

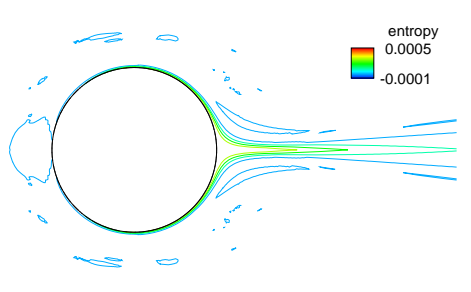

(b) NAGG-IDW

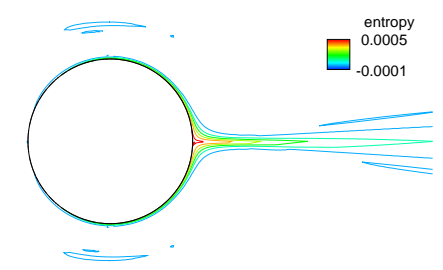

(e) WLSQ(1)-IC

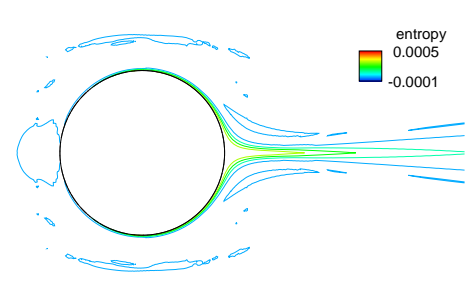

(c) NAGG-PLW

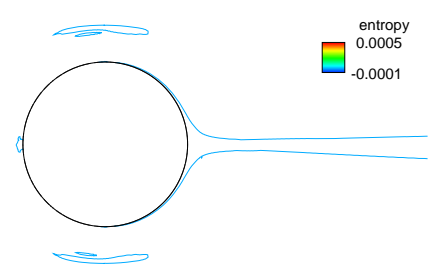

(f) VWLSQ(1-0)-ITER

Fig. 32. Entropy contours around the cylinder: triangle mesh.

that the present iterative strategy is only necessary for a small number of time steps, and thus the present method does not significantly change the computational effort. Even for unsteady problems, the present method only requires iteration in the boundary cells, and thus extra computational effort is still small. The computational cost of the iteration in each time step is given in Eq.(32).

\subsection{Subsonic inviscid flow around a NACA 0012 airfoil}

In this case a more complicate geometry is applied to further examine the performance of the present method, and the flow conditions are the same as in the previous case. The SLAU scheme [41] is used as the numerical flux function, and the time step of the LU-SGS scheme is calculated based on CFL $=20$. In this case, a hybrid mesh is used in which 198 (circumferential) $\times 39$ (radial) quadrilateral cells are applied around the airfoil and the other area is filled with 24,141 triangular cells, as shown in Fig. 34 .

In this case, generally the present method again provides lower entropy around the airfoil, including the leading edge and the trailing edge (as shown in Fig.35), and the drag calculated by the present method is also smaller (as shown in Table 5). Therefore, the present method can be more accurate and less dissipative. In addition, as discussed in the previous subsection, the present method achieves linear-exactness for boundary face solutions, as well as the boundary cell gradients, resulting more accurate spatial approximation compared with the WLSQ-IC method, which provides linear-exact gradients but doesn't directly take BCs into account. It should be noted again the drag as an integral variable cannot represent the details of numerical simulations, especially on a mesh of a relative complicate geometry. Moreover, as shown in Fig.36, the iterative procedure is only activated at the beginning of the computation, and thus the extra computational effort is limited.

Table 5. Numerical drag coefficients $\left(C_{d}\right)$ of the NACA 0012 airfoil.

\begin{tabular}{ccccccc}
\hline Grid type & CAGG & NAGG-IDW & NAGG-PLW & WLSQ(1)-GC & WLSQ(1)-IC & VWLSQ(1-0)-ITER \\
\hline Hybrid & 0.001457 & 0.001240 & 0.001429 & 0.001168 & 0.001997 & 0.001020 \\
\hline
\end{tabular}




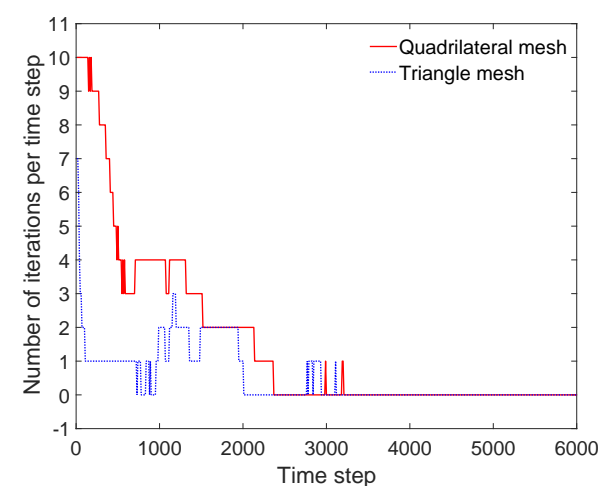

(a) Number of iterations per time step

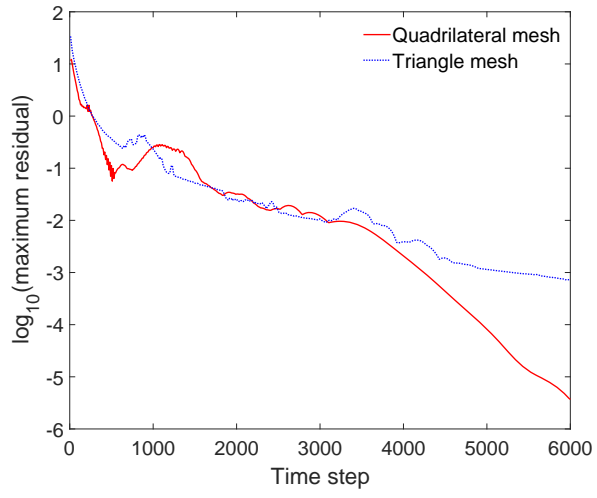

(b) Residual

Fig. 33. The number of iterations per time step of the present method simulating subsonic inviscid flows around a cylinder.

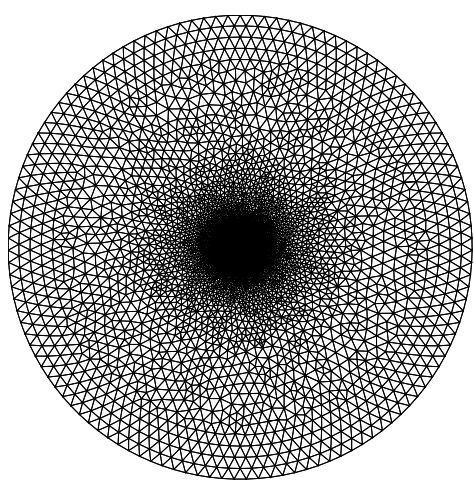

(a) Global view

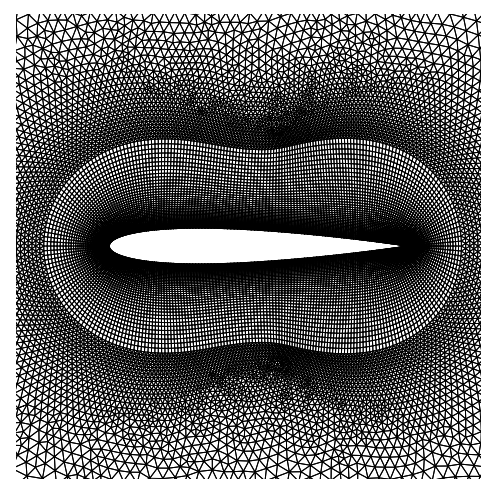

(b) Partial view

Fig. 34. Grids around a NACA 0012 airfoil. 


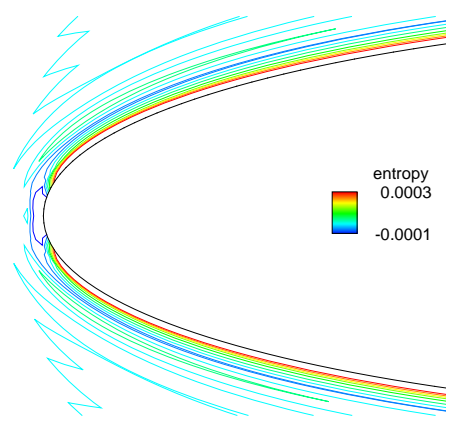

(a) CAGG (leading edge)

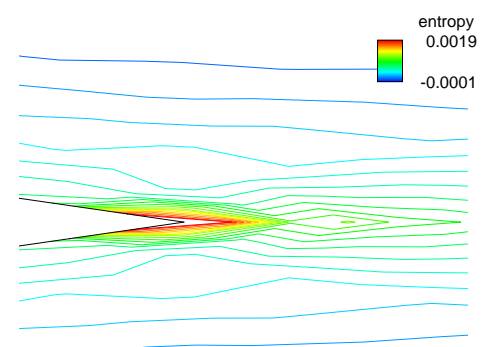

(d) CAGG (trailing edge)

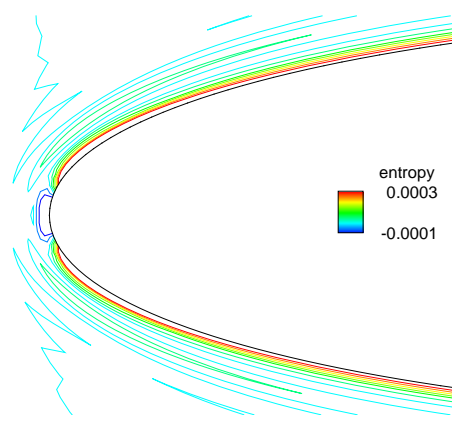

(g) WLSQ(1)-GC (leading edge)

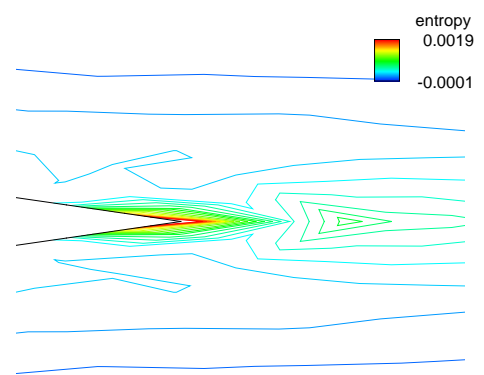

(j) WLSQ(1)-GC (trailing edge)

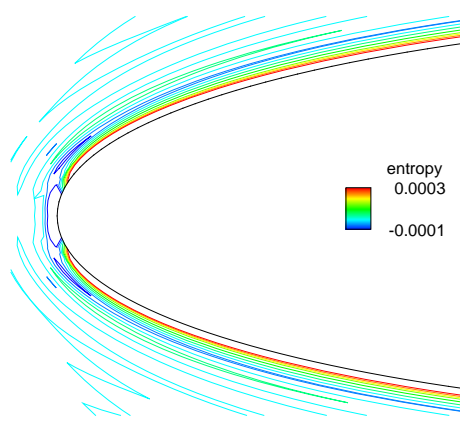

(b) NAGG-IDW (leading edge)

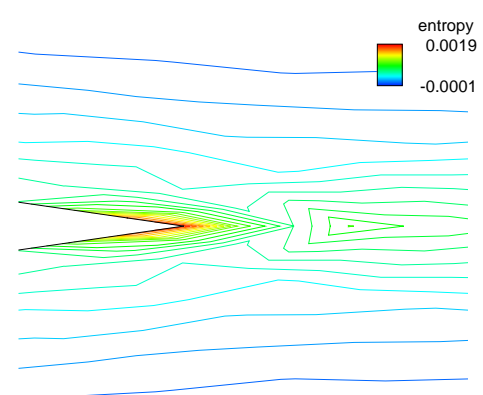

(e) NAGG-IDW (trailing edge)

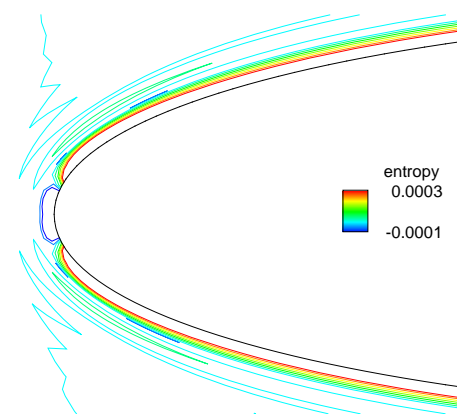

(h) WLSQ(1)-IC (leading edge)

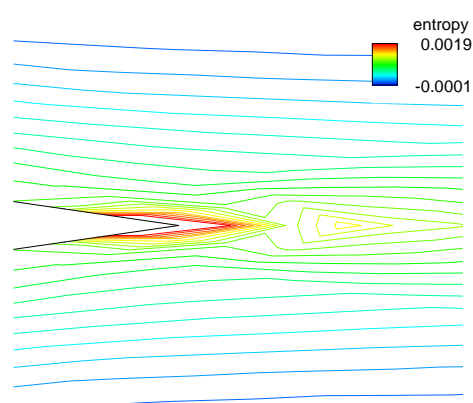

(k) WLSQ(1)-IC (trailing edge)

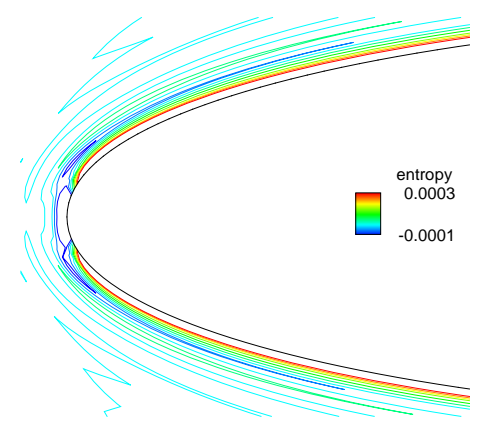

(c) NAGG-PLW (leading edge)

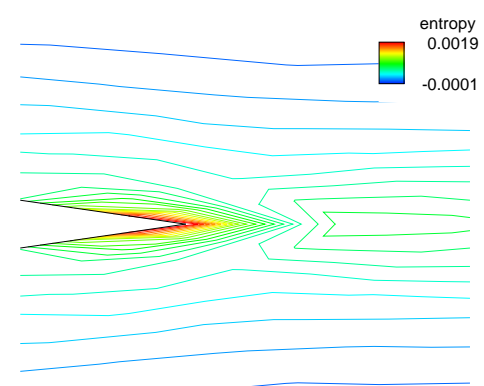

(f) NAGG-PLW (trailing edge)

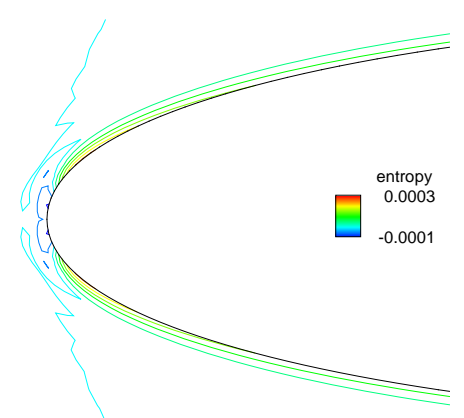

(i) VWLSQ(1-0)-ITER (leading edge)

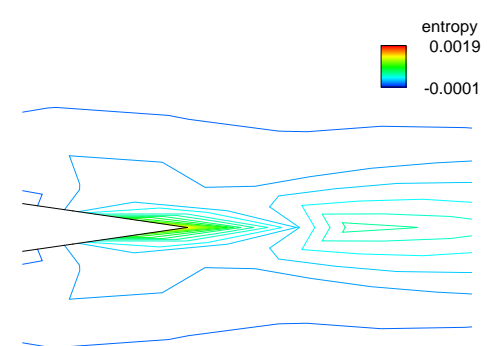

(1) VWLSQ(1-0)-ITER (trailing edge)

Fig. 35. Entropy contours around the NACA 0012 airfoil. 


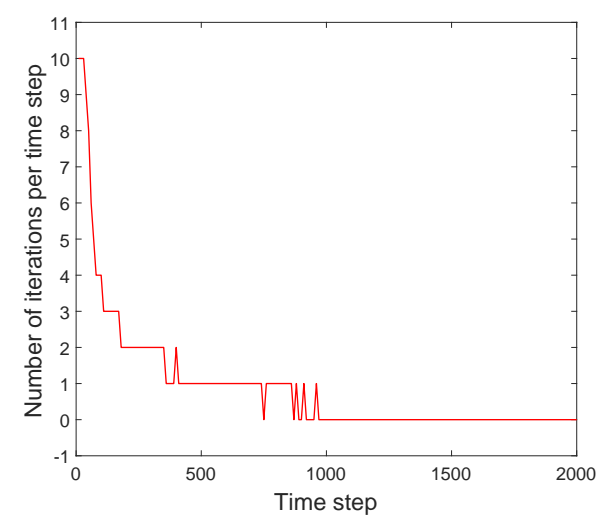

(a) Number of iterations per time step

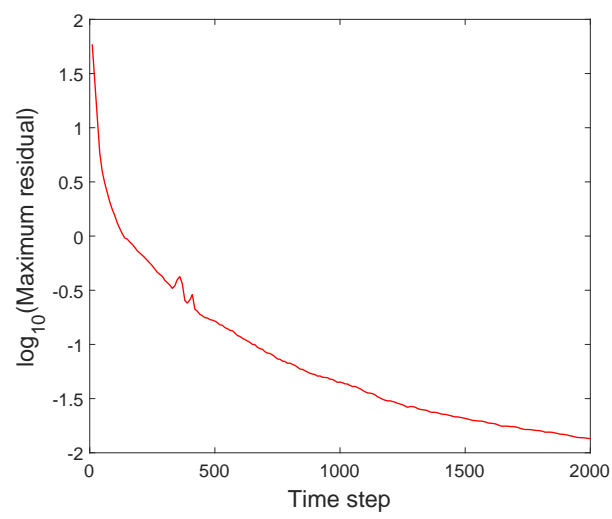

(b) Residual

Fig. 36. The number of iterations per time step of the present method simulating subsonic inviscid flows around a NACA 0012 airfoil.

\subsection{Prandtl-Meyer flow}

The Prandtl-Meyer flow, as shown in Fig.37, is simulated in this subsection to further examine the near boundary performance of the present method. Here the deflection angle $\delta$ of the airflow is set to $20^{\circ}$, and the shadowed region within the red box is used as the computation domain. It can be seen that the upper and lower boundaries of the simulation domain are superposed on streamlines, and thus the slip wall boundary condition is applied. The inlet Mach number is 1.000016 . In this case, a regular triangle mesh having 1800 cells is used for the discretization. The van Leer scheme [38] is used to calculate the numerical flux, and the time step of the LU-SGS scheme is calculated based on $\mathrm{CFL}=100$. The pressure error contours are shown in Fig.38.

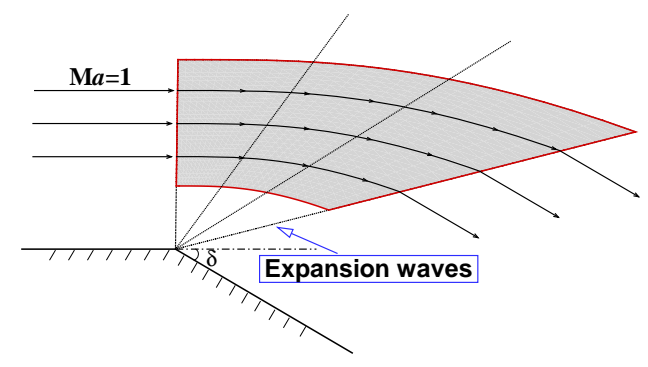

Fig. 37. The schematic of Prandtl-Meyer flow.

As shown in Fig.38(e), the present method almost completely removes the error near boundaries. Whereas, the other methods produce similar error contours. Since the flow Mach number is slightly higher than 1, the numerical error shouldn't be propagating to upstream region, and thus we can conclude that the error are produced due to the inaccurate near-boundary approximations.

\subsection{Inviscid channel flows}

In this subsection, supersonic inviscid flow passing a straight channel is simulated. The free-stream Mach number is given by

$$
M a=2+0.294 \times y
$$

and the angle of attack is 0 . Therefore, the supersonic inlet and outlet BCs are respectively given at left and right boundaries. The slip wall BC is given at the upper and lower boundaries. In this case, the flow field variables are varying linearly, and thus theoretically second-order schemes should be able to provide accurate solutions. This problem is further extended to three-dimension to examine the present method. 


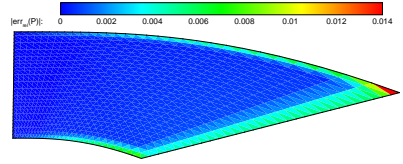

(a) NAGG-IDW

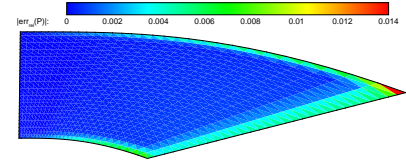

(b) NAGG-PLW

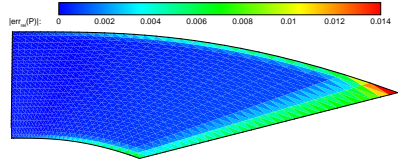

(c) WLSQ(1)-GC

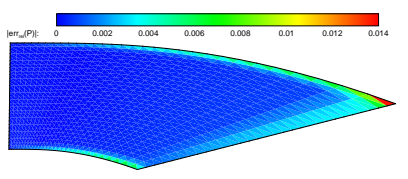

(d) WLSQ(1)-IC

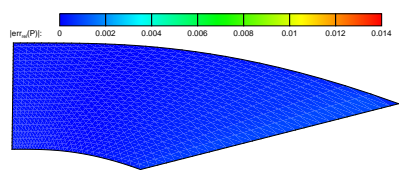

(e) VWLSQ(1-0)-ITER

Fig. 38. Absolute pressure error distribution of the simulations of Prandtl-Meyer flow.

\subsubsection{The two-dimensional case}

For the two-dimensional case, the computational domain is a rectangular box of $[0,2] \times[0,3]$. An irregular triangle mesh having 1200 cells and the corresponding perturbed triangle mesh are used for discretization. The AUSM ${ }^{+}$[40] scheme is used for the convection flux in this case, and the time step of the LU-SGS is calculated based on CFL=1.

The error between the numerical and theoretical velocity in the $x$-direction is shown in Fig.39. It can be found that the result of the NAGG-IDW method at internal cells depends on the grid cell distribution. Namely, its error is smaller on regular triangular grids. Using the PLW method to calculate the nodal solutions significantly improves the internal gradients calculated by the NAGG method, but the near-boundary solutions are not improved. The WLSQ(1)-GC method provides similar result as the NAGG-PLW method. Once again, the near-boundary numerical error is almost completely removed by using the present method, even on the perturbed triangle mesh. It is also interesting to see the difference between the present method and the WLSQ-IC method, which are both linear-exact. The reason causing the error of the WLSQ-IC method is that the boundary face solutions used to calculate the boundary numerical fluxes are not linear-exact, as mentioned in previous subsections. More specifically, along the upper and bottom boundaries, the flow field variables are constant (whereas along the outlet BC the velocity is changing linearly), and thus the error of the WLSQ-IC method becomes zero herein since the other quantities are constant ubiquitously.

In order to show the detail of the approximation, the $x$-direction relative velocity error at the centroid of cells located in $\{x \mid x \in[1.6,1.7]\}$ is extracted from the flow field on the irregular triangle mesh, as shown in Fig.40. It can be found that, the error of the present method is smaller than the other methods. Especially, the present method consistently shows accurate results in boundary cells and internal cells.

\subsubsection{The three-dimensional case}

Here the inviscid linear flow problem discussed in the previous case is extended to three-dimension. The computational domain is a cuboid box of $[0,2] \times[0,3] \times[0,2]$, and the computational domain is discretized to 130640 tetrahedral elements. The velocity is given as Eq.(52), and shown in Fig.41. Similar to the previous 2D case, this 3D case also used the $\mathrm{AUSM}^{+}$scheme to calculate the flux, and the time step of the LU-SGS scheme is calculated based on $\mathrm{CFL}=100$.

The relative velocity errors in the $x$-direction are shown in Fig.42. The errors in the other directions are not shown, since they have similar pattern as in the $x$-direction. The present VWLSQ-ITER method still produces smaller error in this three-dimensional case. The VWLSQ method, which is without the iterative strategy, produces better results than the other traditional methods, but it still produces more error near the boundaries. Therefore, we can conclude that the present VWLSQ-ITER method is also more accurate in simulating this simple three-dimensional problem.

\subsection{A Mach 3 wind tunnel with a step}

In this section we simulate a supersonic unsteady flow with moving shock waves to demonstrate the performance of the proposed method for shock-capturing. The setting of this computation is the same as in Ref.[56]. The length of the channel is 3 , the height is 1 , and a step of relative height 0.2 is located at $x=0.6$. The computational domain is discretized to 26409 triangular cells, and the local mesh close to the corner of the step is shown in Fig.43. 


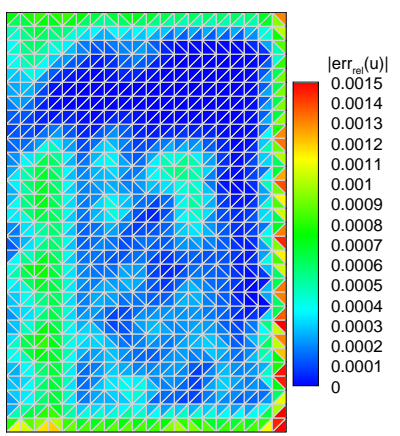

(a) NAGG-IDW (Irregular)

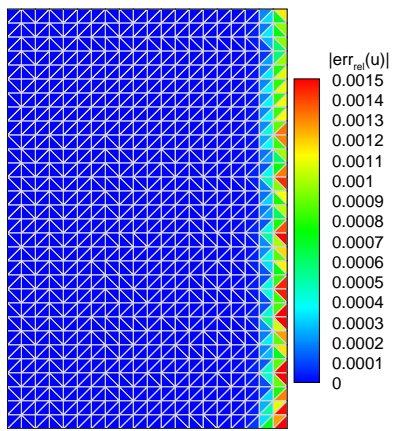

(d) WLSQ(1)-IC (Irregular)

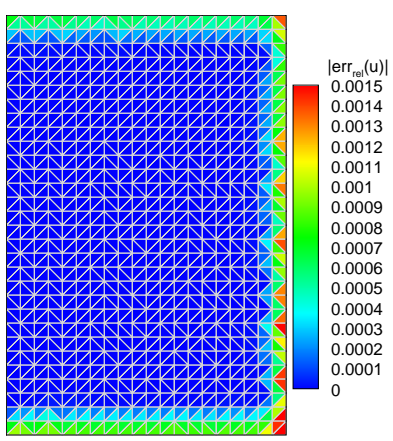

(b) NAGG-PLW (Irregular)

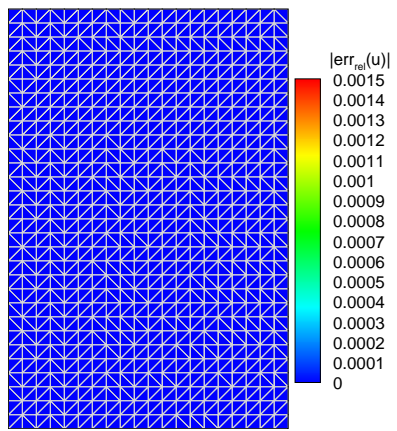

(e) VWLSQ(1-0)-ITER (Irregular)

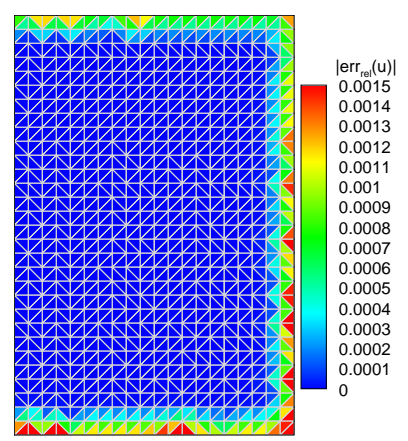

(c) WLSQ(1)-GC (Irregular)

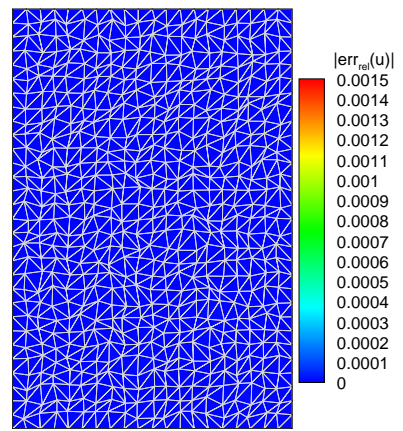

(f) VWLSQ(1-0)-ITER (Perturbed)

Fig. 39. The velocity error contours of the simulations of the two-dimensional inviscid channel flow.

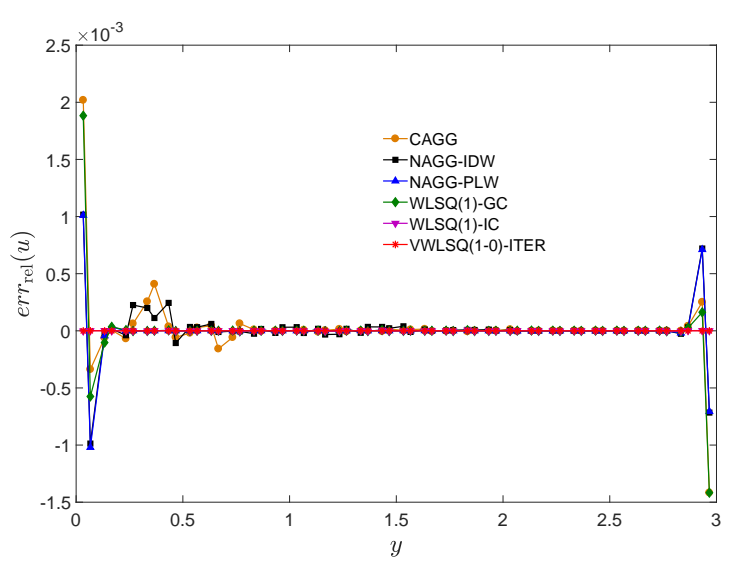

(a) Global view

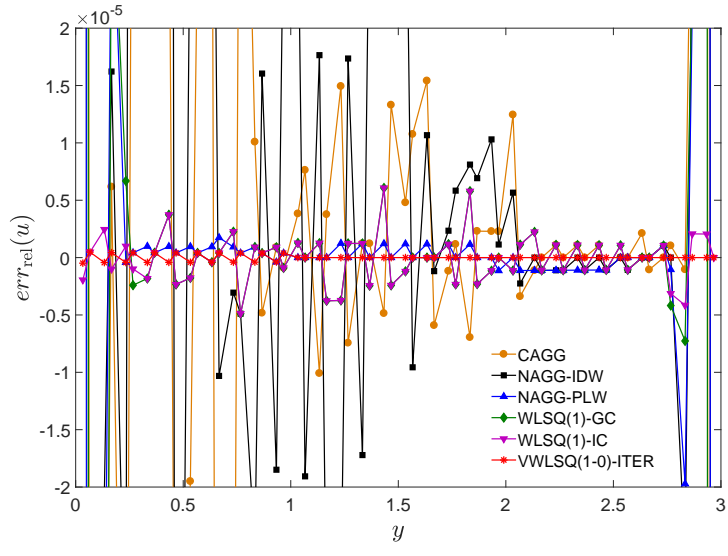

(b) Zoom-in view

Fig. 40. The relative velocity errors in monitor cells of the two-dimensional inviscid channel flow simulation: irregular triangular grid. 


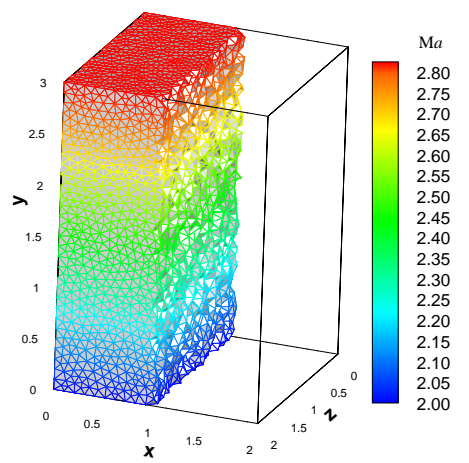

Fig. 41. The exact velocity and the grid of the three-dimensional inviscid channel flow simulation.

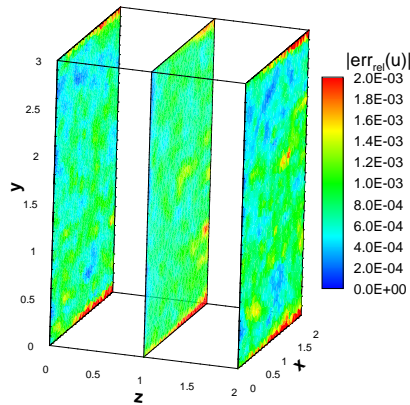

(a) $\mathrm{CAGG}$

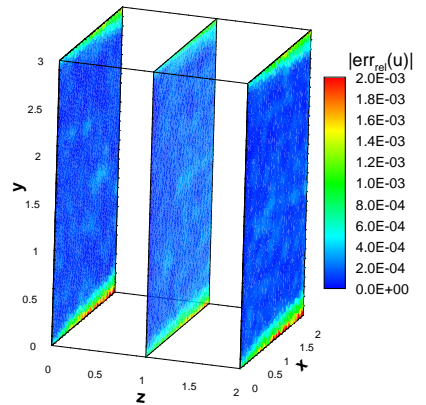

(b) NAGG-IDW

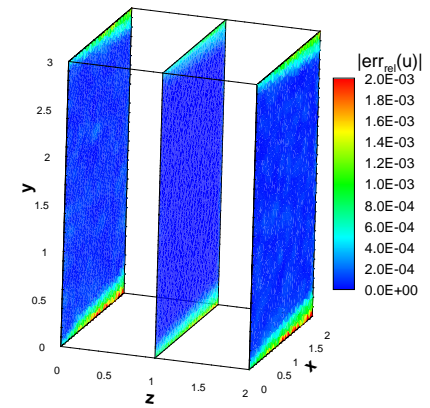

(c) NAGG-PLW

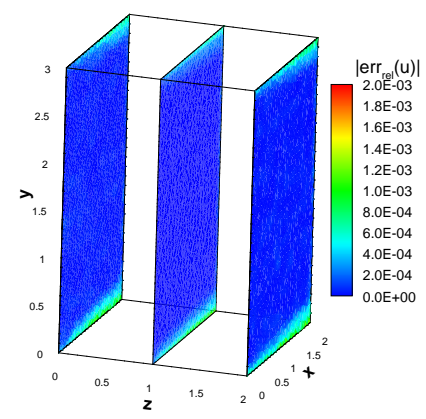

(d) VWLSQ(1-0)

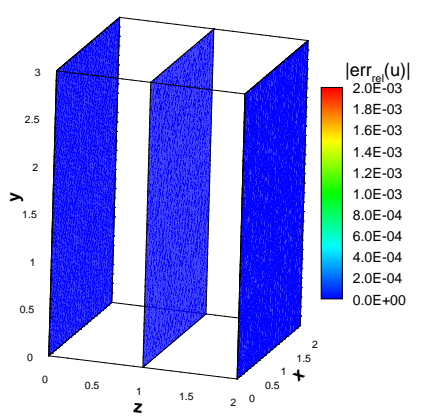

(e) VWLSQ(1-0)-ITER

Fig. 42. The $x$-direction velocity error of the three-dimensional inviscid channel flow simulations. 


\subsubsection{Shock-capturing performance}

The flow Mach number is 3.0, and angle of attack is 0 . At the initial time, a uniform flow field of Mach 3 is set in the channel. The van Leer scheme [38] is used to calculate the flux, and the dual-time stepping [46] with CFL = 10 is used for time advancement, with using LU-SGS for pseudo time solutions. The termination conditions $N_{\mathrm{iter}}^{\max }$ and $\varepsilon$ of the present iterative strategy are respectively defined as 30 and $10^{-8}$. For shock-capturing stability, the MLP-pw limiter [44] with the Venkatakrishnan (denoted as VK) slope limiting function [50] is used $(k=10)$.

The density contours at the dimensionless time $t=4$ are shown in Fig.44. The density distributions in the cross section $y=0.5$ are shown in Fig.45. Since the limiter has strong influence in the numerical results [44], the results of different spatial reconstructions methods are close. However, in Fig.45, the density profile captured by the present method is slightly sharper than those captured by the other methods.

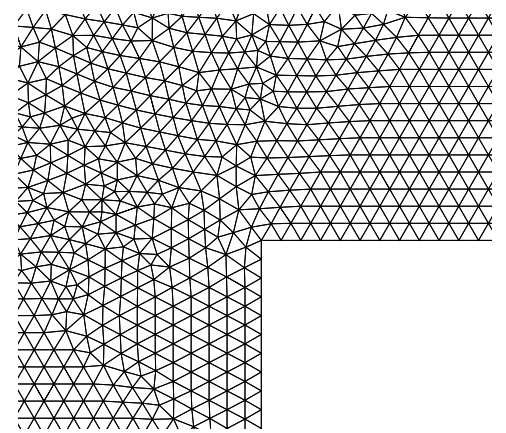

Fig. 43. Local grid near the step corner of the Mach 3 wind tunnel.

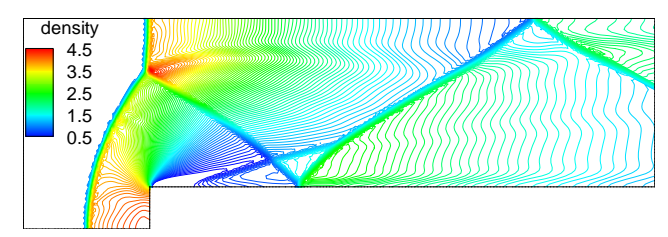

(a) NAGG-IDW

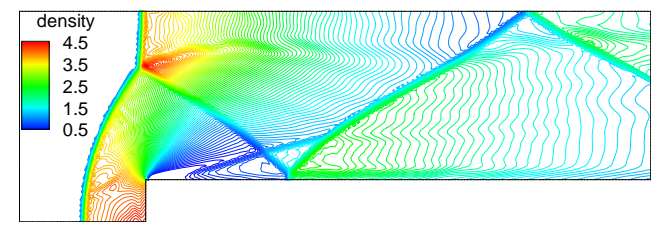

(c) WLSQ(1)-IC

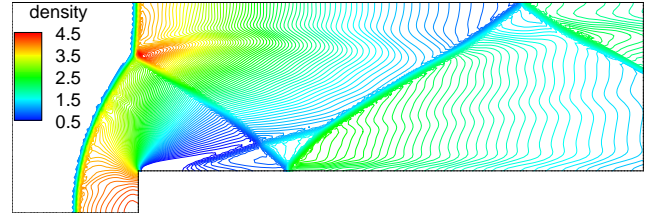

(b) NAGG-PLW

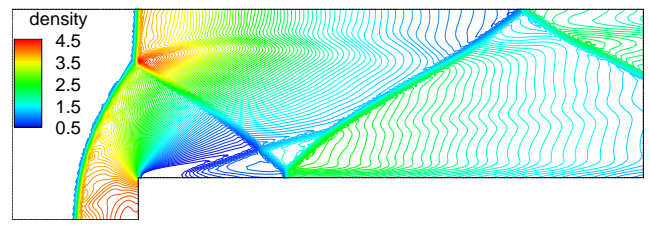

(d) VWLSQ(1-0)-ITER

Fig. 44. Density contours of the Mach 3 wind tunnel flow simulations.

\subsubsection{Limiters for the iterative strategy}

It is worth noted that two limiters are used in the present method while simulating this supersonic case. The first one is the vectorial limiter which is only used in the iterative spatial reconstruction for the near-boundary approximation, and the second one is used for capturing shocks in the whole computation domain, which is actually the conventional idea of using slope limiters and will not be discussed here. The vectorial limiter used in the present VWLSQ-ITER method is usually not invoked if the flow field is smooth and the grid is not highly distorted or curved. Here in this case, shock waves interact with boundaries, and thus it is suitable to assess the performance of using different limiters in the present iterative strategy. Besides the vectorial limiter (denoted as LP) which is recommended, the conventional Barth-Jespersen (denoted as BJ) limiter [20] and the VK limiter are also used in the iterative procedure, to perform the near boundary approximation in a single time step at $t=4$, based on the result shown in Fig.44(d). 


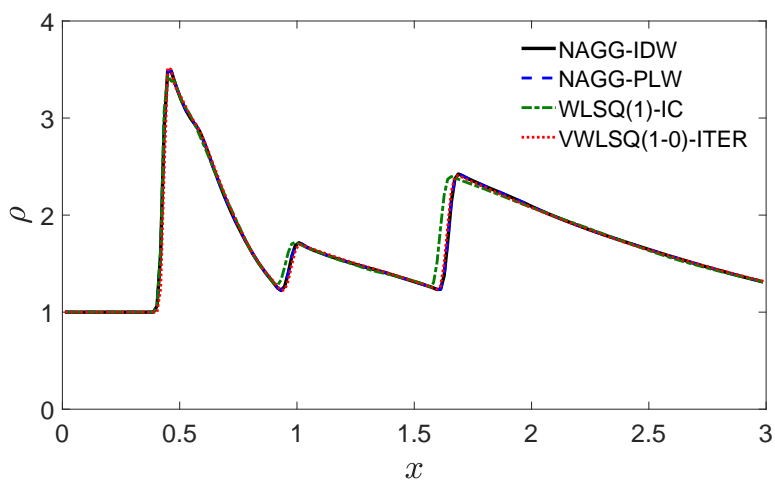

Fig. 45. Density distribution along the cross section $y=0.5$ of the Mach 3 wind tunnel flow simulations.

Five monitor boundary cells are defined, as shown in Fig.46. The density gradients in these cells, which are iteratively calculated, are shown in Table 6 . In the monitor cells, significant changes can be found in the $x$-direction, and thus the limiters should be able to modify the gradient in this direction. Whereas, in the $y$-direction, which is (approximately) parallel to the shock waves, the limitation is expected to be relaxed. It can be found that, a less restrictive $y$-direction component of the gradient, i.e. $\partial \rho / \partial y$, is usually provided by using the vectorial limiter (although the vectorial limiter is usually the most restrictive one in the $x$-direction). Therefore, we may conclude that the present idea of using a vectorial limiter can be helpful for simulating anisotropic flows, reducing unnecessary gradient modifications. In this work, we only investigate inviscid flows. However, the present case can be an evidence of the potential of using the present method for anisotropic viscous flow simulations.

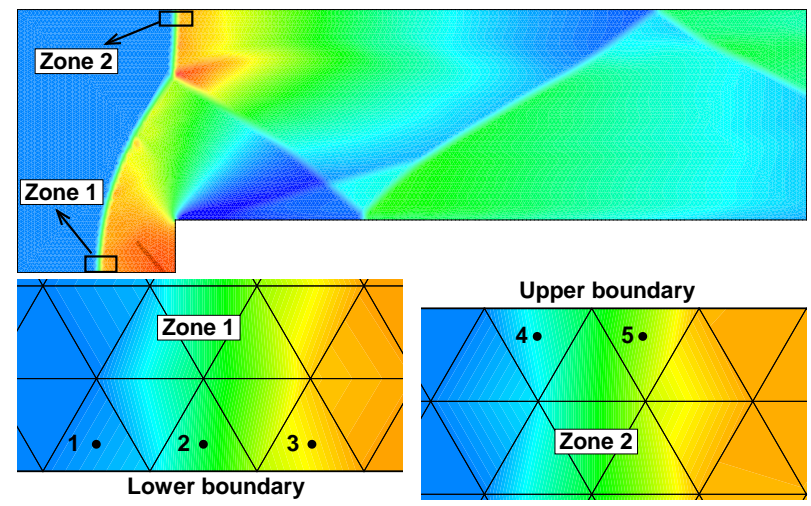

Fig. 46. Monitor boundary cells of the Mach 3 wind tunnel flow simulation.

Table 6. Density gradients in monitor boundary cells calculated by using different limiters in the VWLSQ-ITER method.

\begin{tabular}{|c|c|c|c|c|c|c|c|c|}
\hline \multirow{2}{*}{ Monitor cell } & \multicolumn{4}{|c|}{$\partial \rho / \partial x$} & \multicolumn{4}{|c|}{$\partial \rho / \partial y$} \\
\hline & No limiter & LP & $\mathrm{BJ}$ & VK & No limiter & LP & $\mathrm{BJ}$ & VK \\
\hline 1 & 20.64 & 0.000 & 1.015 & 2.045 & -18.42 & -2.483 & -0.7255 & -1.478 \\
\hline 2 & 117.8 & 119.8 & 120.0 & 120.0 & 9.322 & 8.292 & 8.397 & 8.332 \\
\hline 3 & 50.00 & 0.000 & 1.015 & 1.533 & 25.79 & 1.302 & 0.4148 & 0.6276 \\
\hline 4 & 86.66 & 0.000 & 0.4399 & 0.9818 & 2.644 & 0.7731 & 0.01107 & 0.02546 \\
\hline 5 & 99.10 & 76.19 & 76.82 & 63.31 & -4.037 & -4.265 & -3.187 & -2.540 \\
\hline
\end{tabular}




\section{Conclusions}

Based on the vertex-based WLSQ (VWLSQ) method, we propose an iterative strategy to ensure the linear exactness of the near-boundary spatial reconstruction calculating gradients in boundary cells and solutions at boundary faces. The present method is a subset of implicit gradient reconstruction methods, which were proved to be effective to improve the performance of the second-order FVM, and in the meantime the conventional explicit gradient reconstruction approach is still used in this work for internal cells. Moreover, a vectorial limiter is implemented to ensure that the present spatial reconstruction method is robust for simulations involving discontinuities and highly distorted meshes.

The present iterative strategy updates the solutions at boundary faces and the gradients in boundary cells altogether, until the convergence is achieved. The numerical results of various test cases, involving high-aspect-ratio triangular grids, boundary layer flows (method of manufactured solution) and shock waves, show that the present method provides significant improvements on the near-boundary solution and gradient accuracy, and reduces numerical dissipation in relative practical simulations, e.g. flows around a cylinder or an airfoil. Essentially, the results indicate that the present method is exact for linear functions, which is necessary for the second-order FVM. Moreover, since the iterative strategy is only used for the boundary cells and the iteration convergence can be achieved in a few steps, the extra computational effort is limited, without damaging the overall performance.

It is also worth noted that, at least for the second-order FVM, the error caused by the inaccurate near-boundary approximation may affect the overall solution of a simple problem, e.g. subsonic flows around a cylinder. Therefore, it is necessary to specifically investigate the near-boundary approximation even for second-order FVM schemes.

\section{Acknowledgments}

Funding: This work was financially supported by the National Key Research and Development Program of China (Grant No. 2016YFB0200702) and the National Natural Science Foundation of China (Grant No. 11872144).

Code and data availability: The numerical solver being used is an in-house code that cannot be freely distributed. However, the source code of the implementation of the present methods, as well as the data being shown, can be distributed up on request.

\section{References}

[1] V. A. Titarev, P. Tsoutsanis, D. Drikakis, WENO schemes for mixed-element unstructured meshes, Communications in Computational Physics 8 (2010) 585-609.

[2] P. Tsoutsanis, V. Titarev, D. Drikakis, WENO schemes on arbitrary mixed-element unstructured meshes in three space dimensions, Journal of Computational Physics 230 (2011) 1585 - 1601.

[3] Q. Wang, Y.-X. Ren, J. Pan, W. Li, Compact high order finite volume method on unstructured grids III: Variational reconstruction, Journal of Computational Physics 337 (2017) $1-26$.

[4] A. F. Antoniadis, P. Tsoutsanis, D. Drikakis, Assessment of high-order finite volume methods on unstructured meshes for RANS solutions of aeronautical configurations, Computers \& Fluids 146 (2017) $86-104$.

[5] D. J. Mavriplis, Unstructured mesh discretizations and solvers for computational aerodynamics, in: 18th Computational Fluid Dynamics Conference, AIAA 2007-3955, Miami, FL, 2007. doi:10.2514/6.2007-3955.

[6] B. Cockburn, G. E. Karniadakis, C.-W. Shu (Eds.), Discontinuous Galerkin methods: theory, computation and applications, 1 ed., SpringerVerlag Berlin Heidelberg, 2000. doi:10.1007/978-3-642-59721-3.

[7] H. T. Huynh, A flux reconstruction approach to high-order schemes including discontinuous Galerkin methods, in: 18th AIAA Computational Fluid Dynamics Conference, AIAA Paper 2007-4079, Miami, FL, 2007. doi:10 . 2514/6 . 2007-4079.

[8] B. Diskin, J. Thomas, Accuracy of Gradient Reconstruction on Grids with High Aspect Ratio, Technical Report Report No. 2008-12, LF998199, National Inst. of Aerospace, Hampton, VA, 2008.

[9] B. Diskin, J. L. Thomas, E. J. Nielsen, H. Nishikawa, J. A. White, Comparison of node-centered and cell-centered unstructured finite-volume discretizations: Viscous fluxes, AIAA Journal 48 (2010) 1326-1338.

[10] B. Diskin, J. L. Thomas, Comparison of node-centered and cell-centered unstructured finite-volume discretizations: Inviscid fluxes, AIAA Journal 49 (2011) 836-854.

[11] H. Nishikawa, From hyperbolic diffusion scheme to gradient method: Implicit Green-Gauss gradients for unstructured grids, Journal of Computational Physics 372 (2018) 126-160.

[12] H. Nishikawa, J. A. White, Face-averaged nodal-gradient approach to cell-centered finite-volume method on triangular grids, in: AIAA Aviation 2019 Forum, AIAA 2019-3636, Dallas, Texas, 2019.

[13] H. Nishikawa, Efficient gradient stencils for robust implicit finite-volume solver convergence on distorted grids, Journal of Computational Physics 386 (2019) 486-501.

[14] D. G. Holmes, S. D. Connell, Solution of the 2D Navier-Stokes equations on unstructured adaptive grids, in: 9th Fluid Dynamics Conferences, AIAA 89-1932, Buffalo, NY, 1989. doi:10.2514/6.1989-1932. 
[15] N. T. Frink, P. Parikh, S. Pirzadeh, A fast upwind solver for the Euler equations on three-dimensional unstructured meshes, in: 29th Aerospace Sciences Meetings, AIAA 91-0102, Reno, NV, 1991. doi:10.2514/6.1991-102.

[16] R. D. Rausch, J. T. Batina, H. T. Y. Yang, Spatial adaptation of unstructured meshes for unsteady aerodynamic flow computations, AIAA Journal 30 (1992) 1243-1251.

[17] N. T. Frink, Recent progress toward a three-dimensional unstructured navier-stokes flow solver, in: 32th Aerospace Sciences Meetings and Exhibit, AIAA Paper 94-0061, Reno, NV, 1994. doi:10.2514/6.1994-61.

[18] S. E. Kim, B. Makarov, D. Caraeni, A multi-dimensional linear reconstruction scheme for arbitrary unstructured mesh, in: 16th Computational Fluid Dynamics Conference, AIAA Paper 2003-3990, Orlando, FL, 2003. doi:10 . 2514/6. 2003-3990.

[19] A. Katz, V. Sankaran, High aspect ratio grid effects on the accuracy of navier-stokes solutions on unstructured meshes, Computers \& Fluids 65 (2012) 66-79.

[20] T. J. Barth, D. C. Jespersen, The design and application of upwind schemes on unstructured meshes, in: 27th Aerospace Sciences Meetings, AIAA 89-0366, Reno, NV, 1989. doi:10.2514/6.1989-366.

[21] H. Nishikawa, A face-area-weighted 'centroid' formula for finite-volume method that improves skewness and convergence on triangular grids, Journal of Computational Physics 401 (2020) 109001.

[22] D. J. Mavriplis, Revisiting the least-squares procedure for gradient reconstruction on unstructured meshes, in: 16th Computational Fluid Dynamics Conference, AIAA 2003-3990, Orlando, FL, 2003. doi:10.2514/6.2003-3986.

[23] E. Shima, K. Kitamura, T. Haga, Green-Gauss/weighted-least-squares hybrid gradient reconstruction for arbitrary polyhedra unstructured grids, AIAA Journal 51 (2013) 2740-2747.

[24] F. Zhang, A vertex-weighted-least-squares gradient reconstruction, 2017. ArXiv:1702.04518v1 [physics.flu-dyn].

[25] F. Zhang, Research on Spatial Discretization Schemes for Finite Volume Method on Unstructured Grids, Ph.D. thesis, Dalian University of Technology, Dalian, 2017.

[26] J. A. White, H. Nishikawa, R. A. Baurle, A 3-D nodal-averaged gradient approach for unstructured-grid cell-centered finite-volume methods for application to turbulent hypersonic flow, in: AIAA Scitech 2020 Forum, Orlando, FL, 2020.

[27] M. Berger, M. J. Aftosmis, S. M. Murman, Analysis of slope limiters on irregular grids, in: 43rd AIAA Aerospace Sciences Meeting, AIAA paper 2005-0490, Reno, NV, 2005.

[28] S. May, M. Berger, Two-dimensional slope limiters for finite volume schemes on non-coordinate-aligned meshes, SIAM J. Sci. Comput. 35 (2013) A2163-A2187.

[29] Q. Abbas, J. Nordstrom, Weak versus strong no-slip boundary conditions for the navier-stokes equations, Engineering Applications of Computational Fluid Mechanics 4 (2010) 29-38.

[30] J. Nordstrom, S. Eriksson, P. Eliasson, Weak and strong wall boundary procedures and convergence to steady-state of the navier-stokes equations, Journal of Computational Physics 231 (2012) 4867-4884.

[31] G. Mengaldo, D. De Grazia, F. Witherden, A. Farrington, P. Vincent, S. Sherwin, J. Peiro, A guide to the implementation of boundary conditions in compact high-order methods for compressible aerodynamics, in: 7th AIAA Theoretical Fluid Mechanics Conference, AIAA 2014-2923, Atlanta, GA, 2014. doi:10.2514/6.2014-2923.

[32] H. Nishikawa, Accuracy-preserving boundary flux quadrature for finite-volume discretization on unstructured grids, Journal of Computational Physics 281 (2015) $518-555$.

[33] H. Nishikawa, Y. Nakashima, Dimensional scaling and numerical similarity in hyperbolic method for diffusion, Journal of Computational Physics 355 (2018) 121-143.

[34] A. Jameson, W. Schmidt, E. Turkel, Numerical solution of the euler equations by finite volume methods using runge kutta time stepping schemes, in: 14th fluid and plasma dynamics conference, 1981, p. 1259.

[35] A. Dadone, B. Grossman, A rotated upwind scheme for the euler equations, in: 29th AIAA Aerospace Sciences Meeting, 1991.

[36] A. Dadone, B. Grossman, Surface boundary conditions for the numerical solution of the euler equations, AIAA journal 32 (1994) $285-293$.

[37] H. Lee, J. J. Jeong, H. K. Cho, H. Y. Yoon, An improved numerical scheme to evaluate the pressure gradient on unstructured meshes for two-phase flow analysis, International Communications in Heat and Mass Transfer 37 (2010) 1273-1279.

[38] B. van Leer, Flux-vector splitting for the Euler equations, in: E. Krause (Ed.), Eighth International Conference on Numerical Methods in Fluid Dynamics, volume 170 of Lecture Notes in Physics, Springer Berlin Heidelberg, Aachen, Germany, 1982, pp. 507-512.

[39] E. Toro, M. Spruce, W. Speares, Restoration of the contact surface in the HLL-Riemann solver, Shock Waves 4 (1994) $25-34$.

[40] M.-S. Liou, A sequel to AUSM: AUSM+', Journal of Computational Physics 129 (1996) 364-382.

[41] E. Shima, K. Kitamura, Parameter-free simple low-dissipation AUSM-family scheme for all speeds, AIAA Journal 49 (2011) $1693-1709$.

[42] F. Zhang, J. Liu, B. Chen, W. Zhong, Evaluation of rotated upwind schemes for contact discontinuity and strong shock, Computers \& Fluids 134-135 (2016) 11-22.

[43] F. Zhang, J. Liu, B. Chen, W. Zhong, A robust low-dissipation AUSM-family scheme for numerical shock stability on unstructured grids, Int. J. Numer. Meth. Fluids 84 (2017) 135-151.

[44] F. Zhang, J. Liu, B. Chen, Modified multi-dimensional limiting process with enhanced shock stability on unstructured grids, Computers \& Fluids 161 (2018) $171-188$.

[45] S. Yoon, A. Jameson, Lower-upper symmetric-gauss-seidel method for the euler and navier-stokes equations, AIAA Journal 26 (1988) 1025-1026.

[46] A. Jameson, Time dependent calculations using multigrid, with applications to unsteady flows past airfoils and wings, in: 10th Computational Fluid Dynamics Conference, AIAA Paper 91-1596, Honolulu, HI, 1991.

[47] T. J. Barth, P. . Frederickson, Higher order solution of the Euler equations on unstructured grids using quadratic reconstruction, in: 28th Aerospace Sciences Meeting, AIAA 90-0013, Reno, NV, 1990.

[48] N. T. Frink, Assessment of an unstructured-grid method for predicting 3-d turbulent viscous flows, in: 34th Aerospace Sciences Meeting, AIAA 1996-292, Reno, NV, 1996. doi:10.2514/6.1996-292.

[49] F. Zhang, J. Liu, B. Chen, W. Zhong, Research on vertex variables reconstruction for cell-centered finite volume method, Journal of Dalian University of Technology 55 (2015) 449-456.

[50] V. Venkatakrishnan, Convergence to steady state solutions of the Euler equations on unstructured grids with limiters, Journal of Computational Physics 118 (1995) 120-130. 
[51] L. Chen, R. Li, An integrated linear reconstruction for finite volume scheme on unstructured grids, Journal of Scientific Computing 68 (2016) 1172-1197.

[52] P. J. Roache, Code verification by the method of manufactured solutions, Transactions of the ASME 124 (2002) 4-10.

[53] C. J. Roy, Review of code and solution verification procedures for computational simulation, Journal of Computational Physics 205 (2005) $131-156$.

[54] A. Katz, V. Sankaran, Mesh quality effects on the accuracy of CFD solutions on unstructured meshes, Journal of Computational Physics 230 (2011) 7670-7686.

[55] H. Sun, D. L. Darmofal, R. Haimes, On the impact of triangle shapes for boundary layer problems using high-order finite element discretization, Journal of Computational Physics 231 (2012) 541 - 557.

[56] R. Abgrall, On essentially non-oscillatory schemes on unstructured meshes: Analysis and implementation, Journal of Computational Physics 114 (1994) 45-58. 